ARTICLE

https://doi.org/10.1038/s41467-019-12738-w

\title{
Phagocytosis is mediated by two-dimensional assemblies of the F-BAR protein GAS7
}

Kyoko Hanawa-Suetsugu'1,10, Yuzuru Itoh 2,8,10, Maisarah Ab Fatah ${ }^{1,10}$, Tamako Nishimura ${ }^{1,10}$, Kazuhiro Takemura ${ }^{3}$, Kohei Takeshita4, Satoru Kubota', Naoyuki Miyazaki ${ }^{5}$, Wan Nurul Izzati Wan Mohamad Noor', Takehiko Inaba', Nhung Thi Hong Nguyen', Sayaka Hamada-Nakahara², Kayoko Oono-Yakura', Masashi Tachikawa6, Kenji Iwasaki (10,9, Daisuke Kohda (1] ${ }^{4,7}$, Masaki Yamamoto ${ }^{4}$, Akio Kitao (i] ${ }^{3}$, Atsushi Shimada (i] ${ }^{4,7 \star ~ \& ~}$ Shiro Suetsugu (iD) ${ }^{1 \star}$

Phagocytosis is a cellular process for internalization of micron-sized large particles including pathogens. The Bin-Amphiphysin-Rvs167 (BAR) domain proteins, including the FCH-BAR (F$B A R$ ) domain proteins, impose specific morphologies on lipid membranes. Most BAR domain proteins are thought to form membrane invaginations or protrusions by assembling into helical submicron-diameter filaments, such as on clathrin-coated pits, caveolae, and filopodia. However, the mechanism by which BAR domain proteins assemble into micron-scale phagocytic cups was unclear. Here, we show that the two-dimensional sheet-like assembly of Growth Arrest-Specific 7 (GAS7) plays a critical role in phagocytic cup formation in macrophages. GAS7 has the F-BAR domain that possesses unique hydrophilic loops for twodimensional sheet formation on flat membranes. Super-resolution microscopy reveals the similar assemblies of GAS7 on phagocytic cups and liposomes. The mutations of the loops abolishes both the membrane localization of GAS7 and phagocytosis. Thus, the sheet-like assembly of GAS7 plays a significant role in phagocytosis.

\footnotetext{
${ }^{1}$ Nara Institute of Science and Technology, Ikoma 630-0192, Japan. ${ }^{2}$ University of Tokyo, Tokyo 113-0032, Japan. ${ }^{3}$ School of Life Science and Technology, Tokyo Institute of Technology, Tokyo 152-8550, Japan. ${ }^{4}$ RIKEN SPring-8 Center, Sayo, Hyogo 679-5148, Japan. ${ }^{5}$ Institute for Protein Research, Osaka University, Suita, Osaka 565-0871, Japan. ${ }^{6}$ Theoretical Biology Laboratory, RIKEN, Wako 351-0198, Japan. ${ }^{7}$ Division of Structural Biology, Medical Institute of Bioregulation, Kyushu University, Fukuoka 812-8582, Japan. ${ }^{8}$ Present address: Science for Life Laboratory, Department of Biochemistry and Biophysics, Stockholm University, Stockholm, Sweden. ${ }^{9}$ Present address: Tsukuba Advanced Research Alliance, Life Science Center for Survival Dynamics, University of Tsukuba, Tsukuba, Japan. ${ }^{10}$ These authors contributed equally: Kyoko Hanawa-Suetsugu, Yuzuru Itoh, Maisarah Ab Fatah, Tamako Nishimura.

*email: ashimada@bioreg.kyushu-u.ac.jp; suetsugu@bs.naist.jp
} 
P hagocytosis is a process that internalizes larger, micron-sized particles than those internalized by other endocytic pathways, such as clathrin-mediated endocytosis ${ }^{1-3}$. The plasma membrane extends and engulfs phagocytic particles. The proteins of the BAR domains have a more rigid membrane-binding surface than the membrane itself, and the architecture of this surface, that is, concave or convex, reflects the mechanisms for membrane curvature generation ${ }^{4}$. The BAR domain proteins with concave membrane-binding surfaces are classified as the $(\mathrm{N}-)$ BAR and FBAR domain subfamilies and function in plasma membrane invaginations with submicron diameters, including clathrin-coated pits and caveola $e^{5-8}$. In contrast, the I-BAR domains with convex surfaces, such as that of IRSp53, function to create protrusions including filopodia ${ }^{9}$. Most BAR, F-BAR and I-BAR domains are thought to form membrane invaginations or protrusions by assembling into submicron-diameter filaments ${ }^{10-12}$. However, the means by which the BAR domain superfamily proteins assemble into micron-scale structures on phagocytic cups has remained unclear.

GAS7 is expressed in the brain, spleen, lung, testis ${ }^{13-15}$ and immune cells, including macrophages, which are capable of phagocytosis ${ }^{16,17}$. GAS7 regulates the formation of membrane protrusions in neurons ${ }^{13,18}$ and invasive lung cancer cells ${ }^{14}$. Some receptors involved in phagocytosis are co-expressed with GAS7 according to Coxpres DB, a database of gene-expression correlations ${ }^{19}$. However, thus far, the function of GAS7 in macrophages, cells highly capable of phagocytosis, has not been studied.

In this study, we show that GAS7 plays a critical role in phagocytic cup formation in macrophages. Crystallographic, electron microscopic, biochemical and cellular localization analyses revealed that the GAS7 F-BAR domain possesses unique hydrophilic loops that contribute to two-dimensional sheet formation of GAS7 on flat membranes of phagocytotic cup, indicating that the sheet-like assembly of GAS7 is essential for phagocytosis.

\section{Results}

GAS7 splicing isoforms. To assess the possible involvement of GAS7 in phagocytosis, we examined the isoforms of GAS7 expressed in macrophages by comparing the molecular weights of various expressed, non-tagged GAS7 isoforms in HeLa cells to that of the endogenous GAS7 in RAW264.7 macrophages. GAS7b (Mus musculus and Homo sapiens), GAS7cb (Mus musculus), and GAS7c (Homo sapiens) are GAS7 splicing isoforms (Supplementary Fig. 1a). In addition to the F-BAR domain, GAS7b possess a WW domain, and GAS7cb and GAS7c possesses a WW and an SH3 domain, while GAS7d contains only the F-BAR domain. As determined by western blotting, HeLa cells do not express endogenous GAS7, while GAS7b is highly expressed in RAW264.7 macrophages (Supplementary Fig. 1b, c).

Two-dimensional assembly of GAS7 on the membrane in vitro. The F-BAR domains often bind to negatively charged membranes, where the negative charge is provided by phosphatidylserine (PS) and phosphoinositides, including phosphatidylinositol $(3,4,5)$-trisphosphate $\left(\mathrm{PIP}_{3}\right)$, which are enriched in the phagocytic cups ${ }^{20}$. To examine the membrane binding of GAS7, giant unilamellar vesicle (GUV) liposomes containing PS and $\mathrm{PIP}_{3}$ were incubated with GAS7 tagged with green fluorescent protein (GFP), and GAS7 assembly was observed by fluorescence microscopy (Fig. 1a). The isolated F-BAR domain and GAS7b both assembled on GUVs without prominent membrane deformation or tubulation (Fig. 1a). Occasionally, the F-BAR domain and GAS7b partially covered the surfaces of GUVs. The amount of GAS7 on the partially covered surface increased in a time-dependent manner, suggesting that the GAS7 assembly continued on the GAS7-bound membrane
(Fig. 1b). Interestingly, the regions of GUVs without GAS7 were later completely covered by GAS7, with the progression at the edges of the GAS7 assembly. Therefore, these results suggested that the binding of GAS7 to the membrane occurs at the pre-existing assemblies of GAS7 on the membrane.

To understand GAS7 assembly on the membrane in detail, we prepared flat membranes by forming monolayered phospholipids on the grid for electron microscopic observations ${ }^{21,22}$. We observed GAS7 on lipid monolayers by electron microscopy (Fig. 1c). The monolayers showed the striations formed by the GAS7 F-BAR domain, GAS7b and GAS7cb, all of which indicated the sheet-like GAS7 assemblies with striations similar to each other on the membrane (Fig. 1c). Consistent with the possible multiple assembly sites of GAS7 on GUVs, there were multiple striations of GAS7 in random directions, suggesting that there are large numbers of GAS7 assemblies on the membrane. The apparent pitches of striation were $\sim 5 \mathrm{~nm}$, as estimated by the Fourier transform of the images (Fig. 1d, e). These striations were not resulted from the membrane because the striation was not observed without protein (Fig. 1c), suggesting the higher order assembly of GAS7 by its F-BAR domain, as shown for FBP17 and CIP4 on the flat membrane ${ }^{10}$.

Structure of the GAS7 F-BAR domain. To understand the functions of GAS7, we determined the crystal structure of the GAS7 F-BAR domain, using the crystals of an F-BAR fragment and GAS7cb (Fig. 2a, Supplementary Fig. 1d, Supplementary Table 1). In the crystals of GAS7cb, only the F-BAR domain was visible in the electron density maps. The structures of the F-BAR domains in both crystals were almost identical, with a root mean square deviation of $1.2 \AA$. The GAS7 F-BAR domain forms a helical-bundle dimer with a shallow concave curvature, which is one of the flattest curvatures amongst the known F-BAR domains (Supplementary Fig. 2a, b).

The asymmetric unit of the F-BAR domain crystal contained two F-BAR dimers. These dimers interacted with their symmetryrelated dimers, forming filamentous oligomers with asymmetric flat surfaces, which we refer to as flat filamentous oligomers (FFOs) (Fig. 2b). The two FFOs formed by the different dimers in the asymmetric unit were essentially identical to each other. The FFOs had a width of $\sim 14 \mathrm{~nm}$ with a $\sim 5 \mathrm{~nm}$ distance between adjacent F-BAR dimers, while the F-BAR dimers were $\sim 22 \mathrm{~nm}$ in length. The lateral alignment of F-BAR domains in FFO appeared to be similar to the pitch of the GAS7 striations on the monolayered membrane (Fig. 1d, e). In the GAS7cb crystals, the periodicity observed on the monolayered membrane appeared to be absent (Supplementary Fig. 2c). Therefore, the GAS7 striations on the membrane might be formed by the assembly of the FFOs.

In CIP4 and FBP17, the contacts between the F-BAR domains in the crystals were consistent with those on the membrane ${ }^{23}$. We hypothesized that the GAS7 filament in the crystal could have physiological relevance, including membrane binding. Therefore, we considered the possible positions of the $\mathrm{WW}$ and $\mathrm{SH} 3$ domains of GAS7 on this filament. The WW and SH3 domains might be positioned on one side of the FFO, because there are huge spaces between the domains for the linker region between the F-BAR and the WW domain to pass through, as shown hypothetically in Fig. 2c.

FFL2 interacts with the membrane without insertion. The striations on the monolayered membrane suggested the possible formation of FFOs, as in the crystal. The FFOs of the F-BAR domain in the crystals appeared to be mediated by two GAS7specific loops, filament forming loop 1 (FFL1) (aa 171-197; GAS7cb amino-acid residue numbers) and filament forming loop 
a

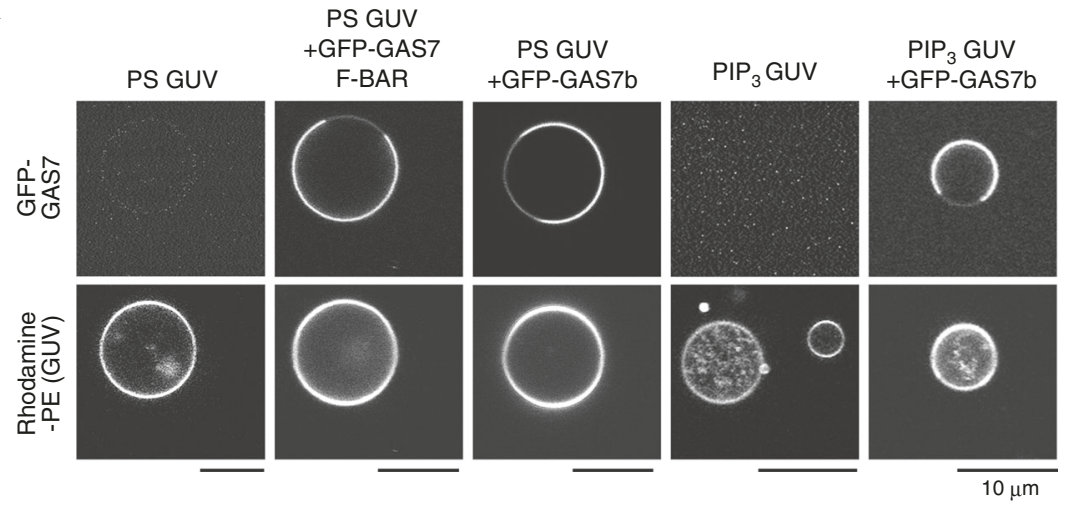

b

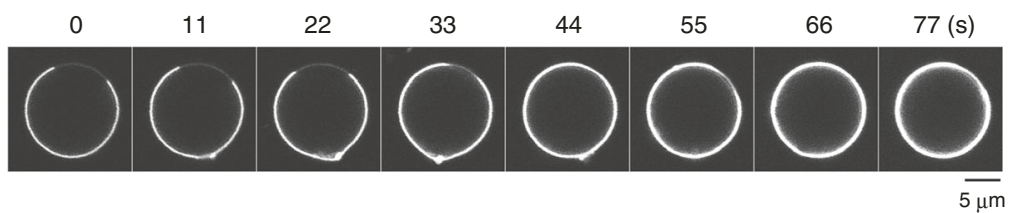

C

GAS7 F-BAR +monolayered membrane

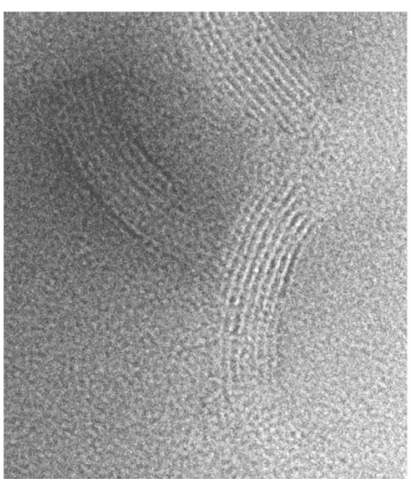

GAS7b D207R

+monolayered membrane

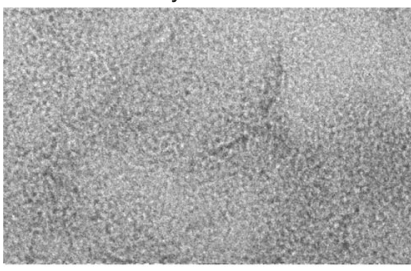

d GAS7 F-BAR + monolayered membrane

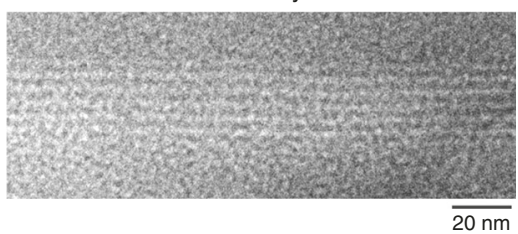

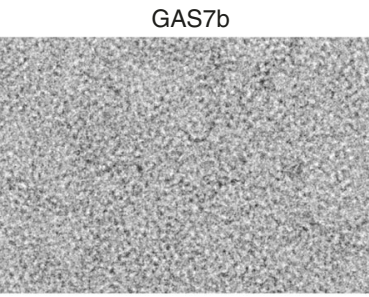

e

GAS7b +monolayered membrane

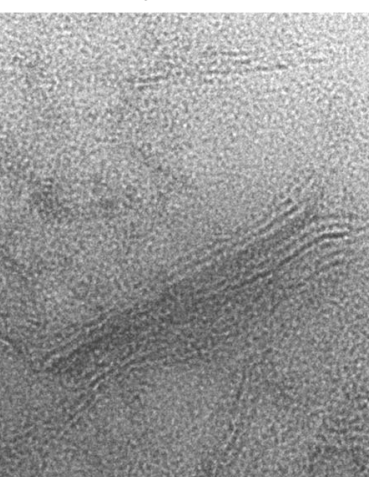

GAS7b
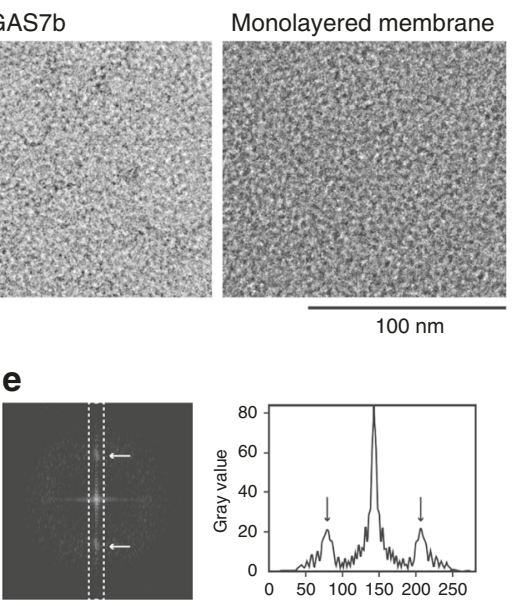

$100 \mathrm{~nm}$

GAS7cb

+monolayered membrane

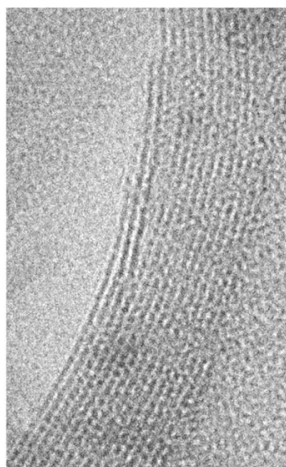

Monolayered membrane

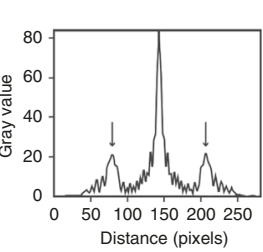

2 (FFL2) (aa 206-219), which are located on the convex and concave surfaces, respectively (Fig. 2a, d, Supplementary Fig. 1d). The positions of the two FFL1s and FFL2s are symmetrical; however, one of the two FFL1s at the convex surface of the dimer interacted with the tip of the adjacent dimer, and one of the two FFL2s at the concave surface of the dimer interacted with a conserved region on the convex surface of the adjacent dimer in the FFO, thus tilting the helix bundle relative to the plane of the
FFO (Fig. 2d, Supplementary Fig. 2d-f). Due to asymmetry, one side of the flat surfaces of the FFO is more positively charged than the other (Fig. 2e). Thus, we supposed that this surface could be the membrane-binding surface. These contacts in the FFO were unique and did not resemble any interactions used for oligomer formation by other BAR, I-BAR and F-BAR domains, including the Pinkbar I-BAR domain, which also forms a planar oligomer (Supplementary Fig. $2 \mathrm{~g}$ ) localized close to the cell-cell contacts ${ }^{24}$. 
Fig. 1 Membrane binding by GAS7 as the flat two-dimensional sheet. a Giant unilamellar vesicles (GUVs) were incubated with 0.5 $\mu$ M GFP-GAS7 F-BAR domain fragment or GAS7b. The proteins were incubated with GUVs containing PC, PE, PS and rhodamine-PE at a molar ratio of 20:20:60:0.02, and GUVs containing PC, PE, PS and PIP 3 at a molar ratio of 40:40:20:5 were observed at $37^{\circ} \mathrm{C}$. Scale bar: $10 \mu \mathrm{m}$. $\mathbf{b}$ The time course of GFP-GAS7b assembly on GUVs. Scale bar: $5 \mu \mathrm{m}$. c Negatively stained transmission electron micrographs of the GAS7 F-BAR domain fragment, GAS7b, GAS7cb and GAS7b D207R mutant on the monolayered membrane. Negatively stained transmission electron micrograph of GAS7b alone and the membrane alone are also shown. Protein samples $(0.1 \mu \mathrm{M})$ were incubated in the presence or absence of lipid monolayers containing PC, PE and PS at a molar ratio of 20:20:60. Scale bar: $100 \mathrm{~nm}$. d Negatively stained transmission electron micrograph of the GAS7 F-BAR domain fragment in the monolayered membrane as in c for e. Scale bar: $20 \mathrm{~nm}$. e The Fourier transform of the micrograph in $\mathbf{d}$. Arrows indicate signals in the reciprocal space corresponding to the periodic striations in $\mathbf{d}$ with the periodic spacing of $\sim 5 \mathrm{~nm}$. A cross-section of the diffraction in the Fourier image is also shown. Arrows indicate the signals showing the regular spacing of $\sim 5 \mathrm{~nm}$

One of the two FFL2s lacks contacts with the adjacent dimer and protrudes from the FFO surface (Supplementary Fig. 2a), suggesting the direct contact of the FFL2 with the membrane. Moreover, the FFL2s are mostly composed of hydrophilic residues (Supplementary Fig. 1d). The membrane binding of GAS7 was then experimentally examined using GAS7b, the isoform highly expressed in macrophages, by a liposome cosedimentation assay. Referring to the other BAR domain studies, we used liposomes reconstituted from bovine brain Folch fraction lipids ${ }^{23,25,26}$, which contained a high amount of PS. GAS7b bound to the PS and $\mathrm{PIP}_{3}$ containing reconstituted liposomes equally well, confirming the relatively low sensitivity to the negatively charged phospholipid species, as also shown by GUVbinding experiments (Fig. 2f, Supplementary Fig. 4a). The replacement of Gln212 at the tip of FFL2 with Arg (Q212R) did not alter the membrane interaction under the physiological salt conditions, but strengthened the membrane binding at a modestly higher salt concentration that reduced the electrostatic interaction (Fig. 2g, h, Supplementary Fig. 4b, c). Given that the introduction of Arg to the tip of FFL2 promoted the membrane binding, it appeared to be unlikely that this loop is inserted into the membrane, as in the case of the 'wedge loop' of the F-BAR domains of PACSINs ${ }^{27,28}$, which contains more hydrophobic residues, especially at the tip of the loop.

To examine the importance of FFL2, we analysed its deletion mutants. The binding of GAS7b $\triangle$ FFL2 mutants to liposomes consisting of the brain Folch fraction was weaker than that of GAS7b (Fig. 2k, Supplementary Fig. 4d). There are several positively charged amino-acid residues on FFL2 (Supplementary Fig. 1d). The replacement of Lys209 with Glu and that of Lys208 and Lys209 with Ala both resulted in reduced binding to liposomes consisting of brain Folch lipids and to reconstituted liposomes containing PS (Fig. 2i, j, Supplementary Fig. 4e, f). These data indicated that the positively charged residues of FFL2 play a crucial role in the membrane binding of GAS7 through electrostatic interactions, again supporting the non-insertion of FFL2 into the membrane.

The above mutants indicated the importance of FFL2 for the membrane binding of GAS7; however, the role of FFL2 in FFO formation was not addressed. The FFLs and tips are the most flexible parts of the F-BAR dimer, as shown by the molecular dynamics (MD) simulation, although their configurations relative to the a-helix bundle did not change during the simulation (Supplementary Fig. 3a, b). Asn177 of FFL1 and Asp207 of FFL2 were in contact with Arg326 on helix a2b (Fig. 2a, Supplementary Fig. 3c), suggesting that these residues stabilized the configuration of the FFLs. The replacement of Asp207 with Arg, which increases the positive charge, was expected to increase the membrane binding simultaneously with the FFL2 destabilization by removing its interaction with the $\alpha$-helix bundle. Interestingly, GAS7b and the D207R mutant exhibited similar binding to reconstituted liposomes containing PS, as examined by a cosedimentation assay (Fig. 21, Supplementary Fig. 4g). To examine the oligomer formation of GAS7, we treated GAS7b with chemical cross-linkers. Highly cross-linked GAS7b was observed after the treatment with chemical cross-linkers as a mobility shift in electrophoresis, which suggested the trimer or tetramer formation by GAS7, although the larger oligomers of GAS7 shown in the FFO model were not easily resolved by electrophoresis (Fig. $2 \mathrm{~m}$ ). In the presence of the liposomes, the increase in the oligomer bands of GAS7b were observed, suggesting the promotion of oligomer formation on the membrane. The D207R mutant exhibited fewer oligomer bands than GAS7b in the electrophoresis after the chemical crosslinking reaction in the presence of the liposomes, suggesting that the oligomerization of the D207R mutant was different from that of GAS7b (Fig. 2m, Supplementary Fig. 4h). Consistently, the striations of D207R mutant on the membrane were different and reduced from those of GAS7b (Fig. 1c). Because the increase of arginine would strengthen the binding to the membrane, the D207R mutation was thought to compensate for the reduction in the oligomerization that would contribute to the membrane binding, suggesting the reason of the membrane binding of the D207R mutant similar to that of GAS7b. Therefore, the GAS7 oligomer formation on the membrane appeared to be promoted over that in the solution, where the configuration of FFL2 was suggested to be important for the adequate oligomerization of GAS7.

The membrane-binding surface of the GAS7 F-BAR domain. The $\mathrm{N}$-surface is perpendicular to the dimer axes, and corresponds to the concave surfaces of the BAR and F-BAR domains and the convex surface of the I-BAR domain (Fig. 2b) ${ }^{29}$. The Nsurface in these domains often contains more conserved residues than those in the other surfaces, and is the membrane-binding surface for most BAR, F-BAR and I-BAR domains ${ }^{23}$. However, the concave $\mathrm{N}$-surface of the GAS7 F-BAR domain dimer is less conserved than the other surfaces (Supplementary Fig. 2d). This is in striking contrast to the other F-BAR domains, such as CIP4 and FBP17, with membrane-binding surfaces containing numerous highly conserved residues ${ }^{23}$. Thus, the $\mathrm{N}$-surface may not be the membrane-binding surface of GAS7, in agreement with the asymmetry of the F-BAR in the FFO model (Fig. 2b).

FFL1 is thought to be distant from the membrane, when membrane binding through the $\mathrm{N}$-surface is assumed. Therefore, we examined the membrane binding of the deletion mutant of FFL1. The binding of the GAS7b $\triangle$ FFL1 mutant to liposomes consisting of brain Folch lipids was weaker than that of GAS7b (Fig. 2g, Supplementary Fig. 4b). This affinity reduction suggested that interaction sites other than the $\mathrm{N}$-surface are involved in the membrane binding of GAS7. Indeed, if we assume the FFO model, then this result is reasonable, as FFL1 is thought to be a critical interaction site for oligomer formation.

The membrane binding of GAS7 is likely to be mediated by electrostatic interactions. Therefore, the positively charged 
a

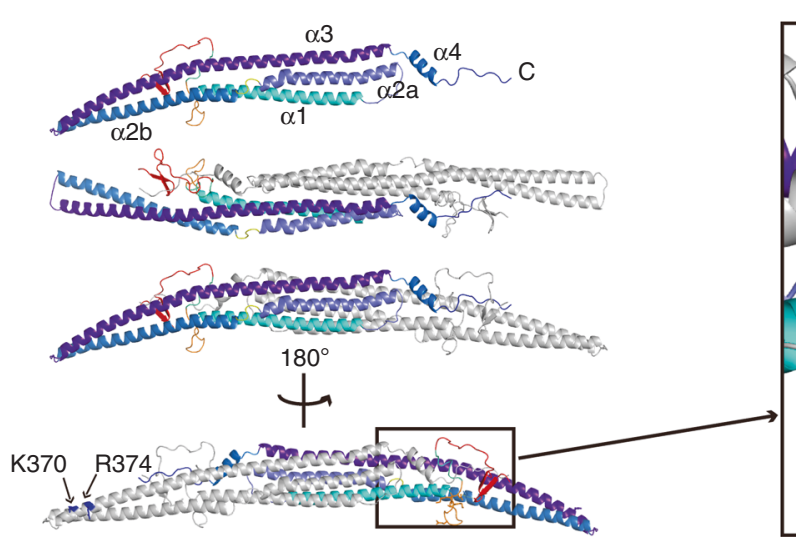

b

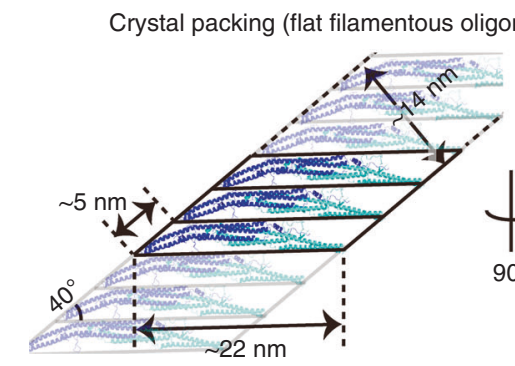

d

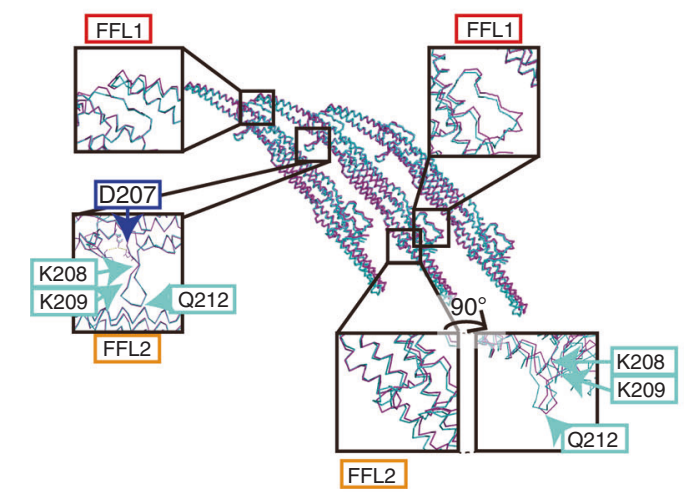

f

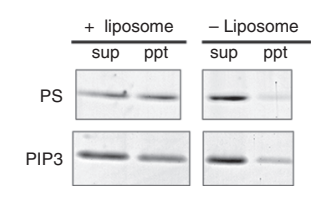

$\mathbf{g}$
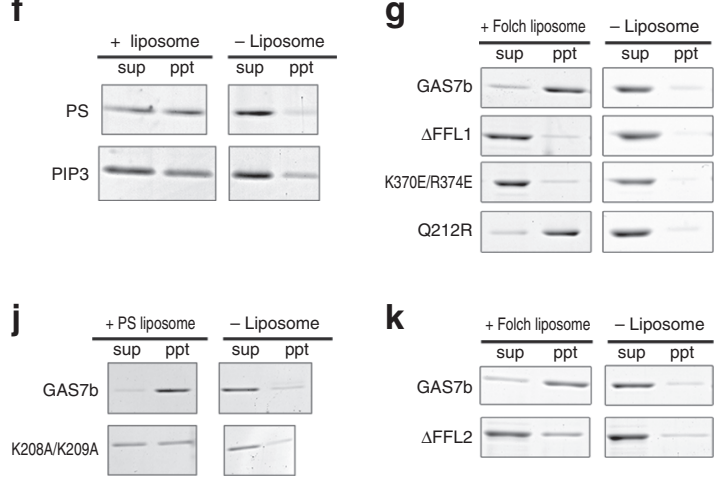

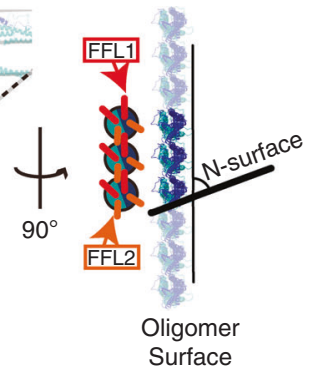

e

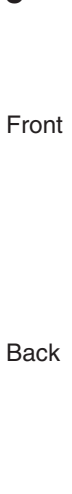

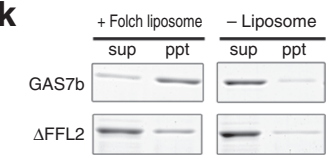

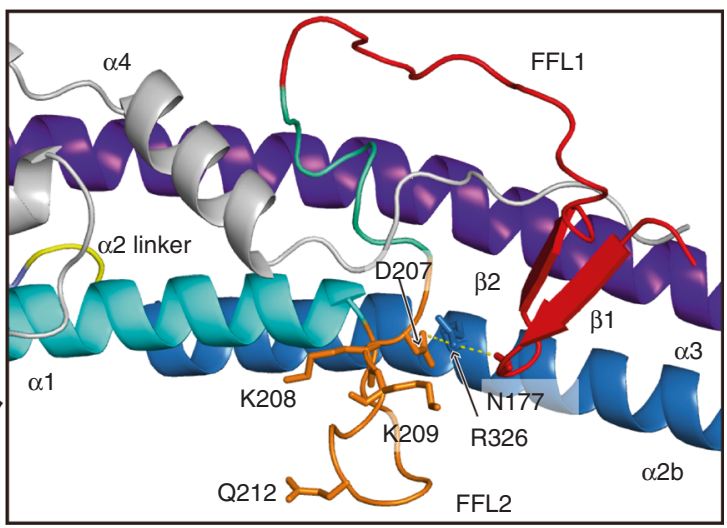

C a hypothetical position of the WW domain

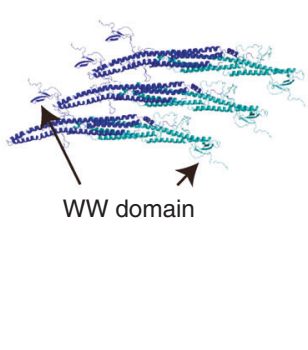

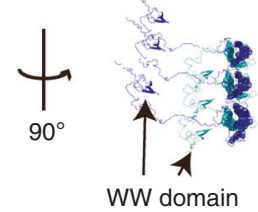

WW domain h

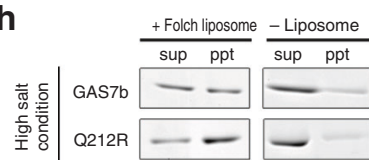

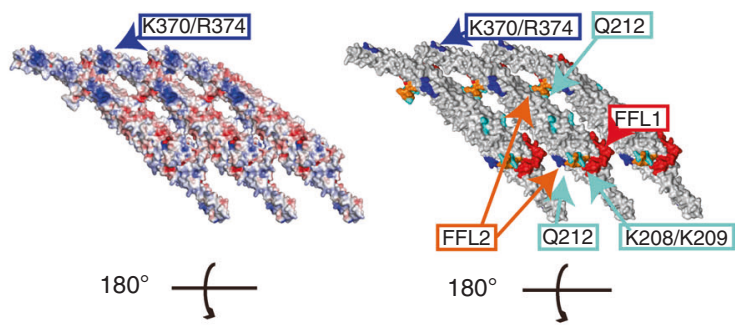

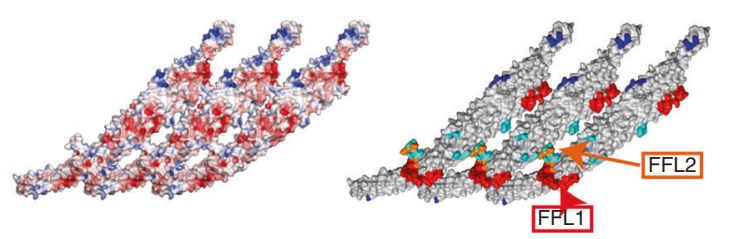

amino-acid residues, Lys370 and Arg374, on the side surface of the dimer, that is, the front surface of the FFO, were examined (Fig. 2a). When these residues were both replaced with glutamate, membrane binding was abolished (Fig. 2g, Supplementary Fig. 4b), further suggesting the membrane binding of GAS7 through the FFO surface.

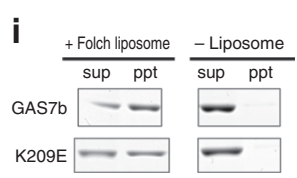

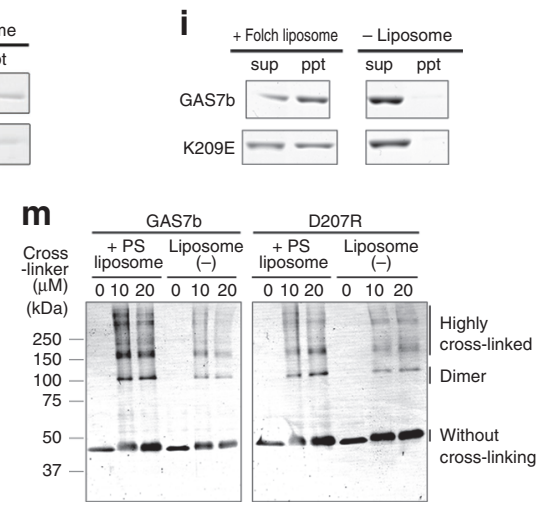


Fig. 2 Crystal structures of GAS7 and the membrane-binding sites. a Ribbon diagrams of the crystal structure of the isolated GAS7 F-BAR domain and the GAS7-specific N-terminal region including FFL1 (red) and FFL2 (orange). Secondary structural elements are indicated. R326 on helix $\alpha 2 b$ contacts N177 on FFL1 as well as D207 on FFL2. The K208, K209, Q212, K370 and R374 residues are also indicated. b Crystal lattice of the isolated F-BAR domain. The asymmetric unit of the crystal contains two F-BAR dimers, which both form the FFO by the crystal packing. The N-surface, which is perpendicular to the dimer axes, is indicated with the illustration of FFL1 and FFL2. c The hypothetical position of the WW domain of GAS7b on the FFO. The two WW domains of the F-BAR dimer can be localized to one side of the FFO. d Superimposition of two FFOs composed of different F-BAR dimers in the asymmetric unit of the crystal (cyan and magenta). The contact sites between the F-BAR domains are indicated. e Surfaces of the FFO. (left) Electrostatic surface potentials of FFOs composed of three F-BAR dimers; blue and red indicate positive and negative charges, respectively. (Right) In the same orientation as in $\mathbf{d}$. The point mutations with defects in the F-BAR domain assembly (blue), and those without defects (cyan) are shown according to Supplementary Fig. 5. FFL1 (red), FFL2 (orange), Q212 (cyan), K208/K209 (cyan) and K370/R374 (blue) are also indicated. f-I GAS7b and its $\Delta$ FFL1, K370E/R374E, Q212R, $\Delta$ FFL2, K209E, K208A/K209A and D207R mutants were examined for their binding to the liposomes by liposome co-sedimentation assays. The presence of proteins in the pellet (ppt) indicates membrane binding. sup: supernatant. The liposomes were of the bovine Folch fraction ( $\mathbf{g}-\mathbf{i}, \mathbf{k}$ ), the PC, PE and PS lipids at a ratio of 20, 20 and $60(\mathbf{f}, \mathbf{j}, \mathbf{I})$, and the PC, PE, PS and PIP lipids at a ratio of 40, 40, 20 and $5(\mathbf{f}) . \mathbf{m}$ Cross-linking of GAS7b and the D207R mutant treated with the $\mathrm{BS}(\mathrm{PEG}) 5$ (PEGylated bis(sulfosuccinimidyl)suberate) cross-linker, in the presence or absence of PS liposomes

assembled into patches with cup-like structures of several microns (Fig. 3a, Supplementary Fig. 5a). The GAS7 localization on the membrane was apparently different from those of other plasma membrane-localized BAR domains with the ability to bind to relatively flat membranes, such as Pinkbar I-BAR and the Nwk ortholog FCHSD1 F-BAR ${ }^{24,30}$ (Supplementary Fig. 5b). Interestingly, the patches excluded the palmitoylated DsRedmonomer (DsRed-membrane), a diffusive plasma membrane marker, suggesting that the patches were sheets composed of highly concentrated GAS7b and GAS7 F-BAR (Fig. 3a, Supplementary Fig. 5a). The patch formation was abolished by the membrane-binding deficient $\triangle \mathrm{FFL} 1, \Delta \mathrm{FFL} 2$ and K370E/R374E mutations, whereas the membrane-binding $\mathrm{Q} 212 \mathrm{R}$ mutant formed patches (Fig. 3a, Supplementary Fig. 5a). The D207R mutation abolished patch formation (Fig. 3a), which was thought to be consistent with the altered oligomer formation in vitro (Fig. 2m). Thus, the defect in the FFL2-mediated oligomer formation is thought to be essential for patch formation.

We also mutated the other positively charged residues. The $\mathrm{K} 312 \mathrm{E} / \mathrm{K} 313 \mathrm{E}$ and $\mathrm{K} 316 \mathrm{E} / \mathrm{K} 317 \mathrm{E}$ mutants did not exhibit the assembly, in contrast to the K279E/K280E and K449E/K450E mutants (Supplementary Fig. 5a). The effective mutations are located between the side and the N-surface of the F-BAR domain dimer structure, suggesting that the FFO surface or a similar one is the membrane-binding surface (Fig. 2e, Supplementary Fig. 5a).

GAS7b assembly on the plasma membrane for phagocytosis. In HeLa cells, the GAS7 F-BAR domain fragment patches sometimes showed invaginations with micro-size diameters, suggesting the possible membrane deformation by the F-BAR domain (Supplementary Fig. 5a). When GAS7b was expressed and observed in live cells, the GAS7b patches appeared at ruffled membranes (Fig. 3b) and eventually transformed into holes reminiscent of phagocytosis (Fig. 3c). Therefore, we examined the localization of GAS7b in RAW264.7 macrophages. An incubation with zymosan, a phagocytic substrate derived from a yeast protein-carbohydrate complex, induced phagocytic cup formation with localized GFPtagged GAS7b (Fig. 3d), which was expressed in GAS7-knockout RAW264.7 macrophages at a similar level to the endogenous GAS7b (Supplementary Fig. 6). Immunofluorescence microscopy revealed the accumulation of endogenous GAS7b with actin filaments at phagocytic cups surrounding the zymosan particles (Fig. 3e). N-WASP and Arp3, a binding partner of GAS7 for actin polymerization and a subunit of the Arp $2 / 3$ complex responsible for the N-WASP-mediated actin polymerization ${ }^{13,14}$, colocalized with GAS7b (Fig. 3f, g). Receptors for phagocytosis, such as complement receptor CR3 component CD11 $\mathrm{b}^{31}$ and mannose receptor CD206 ${ }^{32}$, also colocalized with GAS7b at phagocytic cups (Supplementary Fig. 7a, b).
The phagocytic activity of the RAW264.7 macrophage cells with the reduced expression or knockout of GAS7 was examined, using zymosan. The zymosan uptake was reduced in both GAS7 small interfering RNA (siRNA)-treated and GAS7-knockout cells (Supplementary Fig. 7c-e). IgG-coated bead uptake was also reduced in GAS7-knockout cells (Supplementary Fig. 7f). Consistent with the localization of GAS7 at lamellipodia, the GAS7knockout cells were defective in lamellipodia formation (Supplementary Fig. $7 \mathrm{~g}, \mathrm{~h}$ ). The zymosan phagocytosis was rescued by the endogenous-level forced expression of GAS7b and GAS7cb in the knockout cells, but not by the GAS7b $\triangle$ FFL2 mutant or the F-BAR domain fragments (Fig. 3h, i, Supplementary Fig. 7i). Neither the D207R nor K209E mutant restored phagocytosis, indicating that the oligomerization and the membrane binding of GAS7 were essential for the phagocytic cup formation (Fig. 3h, j).

Similar GAS7b assemblies on membrane in vitro and in cells. The assemblies of GAS7b on GUVs, in HeLa cells, and in macrophage cells were compared by fluorescence recovery after photobleaching (FRAP). On the GUVs, both the F-BAR domain fragment and GAS7b exhibited slow turnovers, although GAS7b exhibited slightly faster recovery after photobleaching (Fig. 3k, Supplementary Fig. 8a, b). Similarly, the GAS7b within a patch in HeLa cells exhibited slower turnover than the GAS7b in the cytosol, but the turnover of the F-BAR domain fragment was slower than that of the GAS7b in a patch (Fig. 31, Supplementary Fig. 8d-f). Interestingly, the GAS7b in the phagocytic cups of macrophages exhibited a similar turnover to that in the GAS7b patches formed in HeLa cells, suggesting similar GAS7b assemblies in these cells (Fig. 31, Supplementary Fig. 8c, d).

Next, the assembly of GAS7b at the single-molecule resolution on membranes was visualized and compared with a simulated GAS7b localization (Fig. 4a). The localization was determined using GAS7b tagged with mEOS4b, a photoconvertible fluorescent protein that enables stochastic observations for super-resolution imaging $^{33}$. The mEOS4b-GAS7b bound to GUVs (Fig. 4b, c) became localized to phagocytic cups, and rescued phagocytosis in GAS7-knockout cells (Fig. 4d, e, Supplementary Fig. 7i). GAS7b assembled into sheets, possibly by the alignment of the FFOs on the lipid monolayer (Fig. 1c). Thus, we created a model of the GAS7b sheet that resembles the electron microscopic observations of the lateral alignment of the FFOs (Fig. 4a). The assembly of mEOS4b-GAS7b was then examined by comparing the occurrence of neighbouring molecules, with random localizations and simulated FFO sheets (Fig. 4a). The occurrences of the observed neighbours of GAS7b on liposomes and on the top of phagocytic cups were similar to each other, and more frequent than those of the non-specifically adsorbed GAS7b on the glass and the bottoms of the phagocytic cups (Fig. 4f-i). Furthermore, the GAS7b 

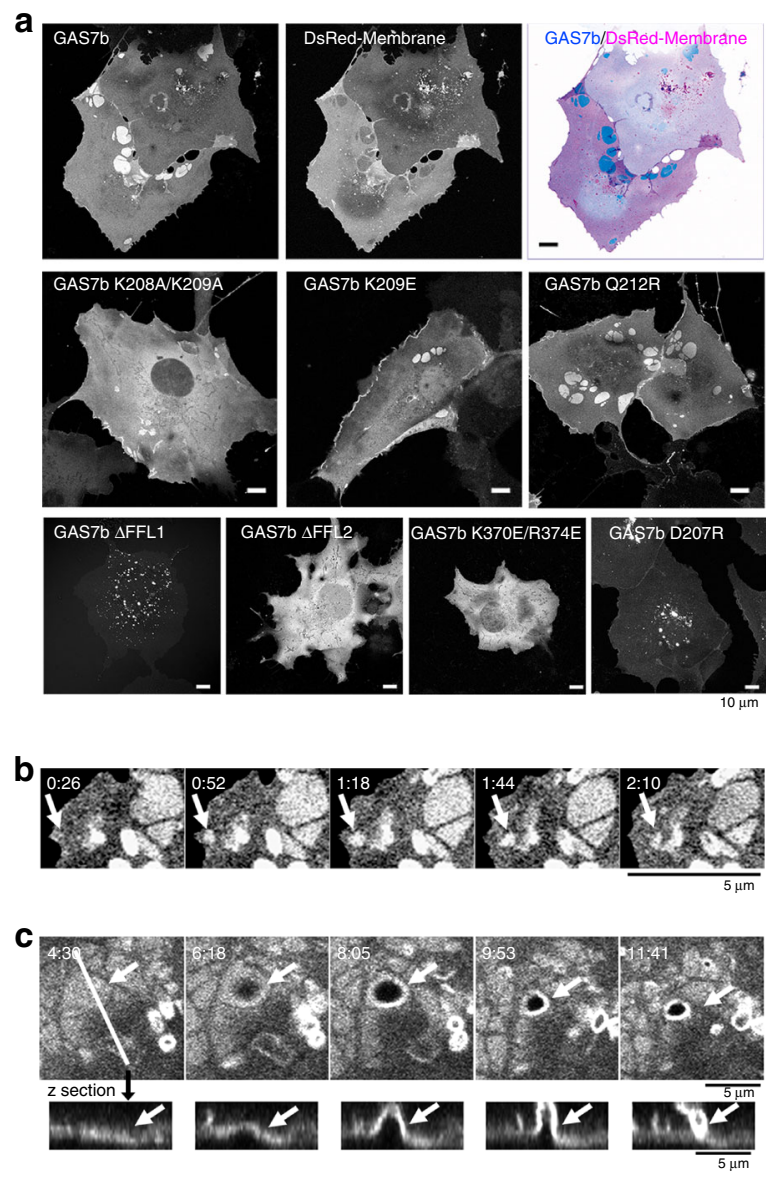

d

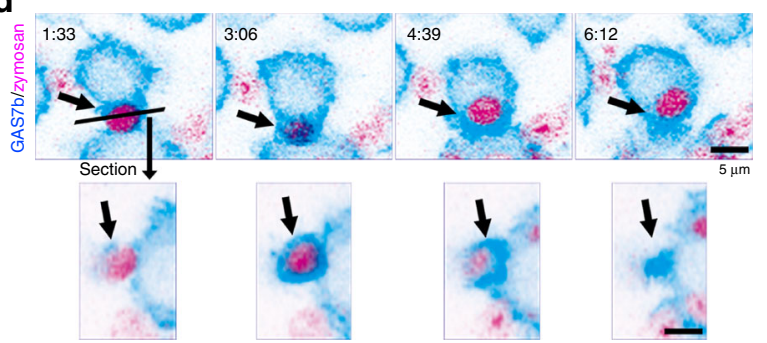

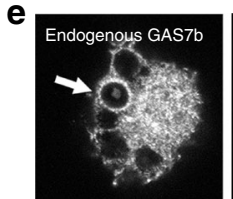
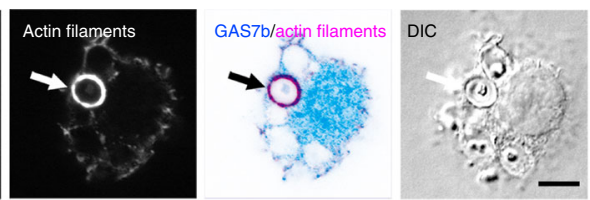

f GAS7b
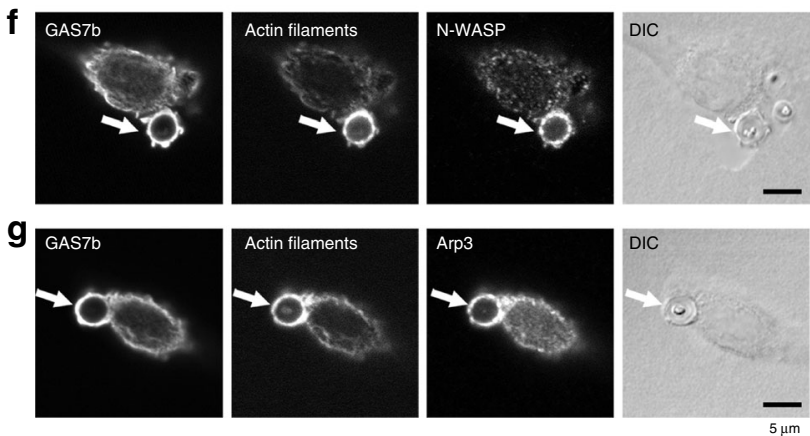

h
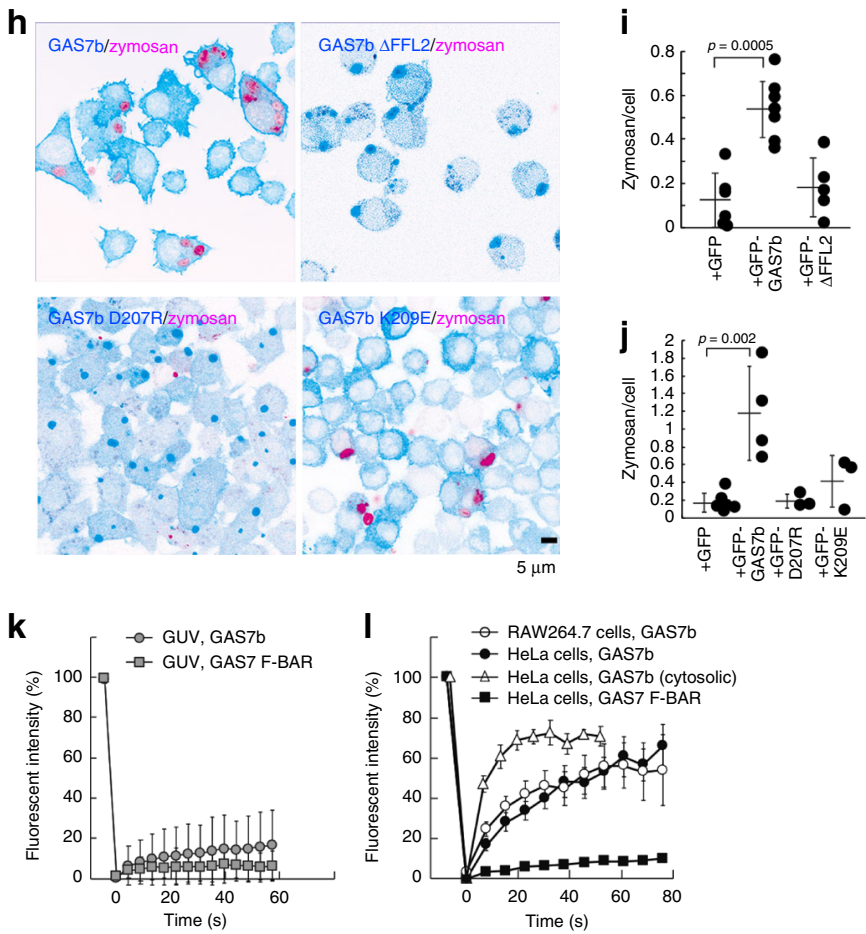

Fig. 3 GAS7b sheet formation in HeLa cells and macrophages. a GFP-GAS7b and its $\Delta$ FFL1, $\Delta$ FFL2, D207R, K208A/K209A, K209E, Q212R and K370E/ R374E mutants expressed in HeLa cells. Plasma membranes of the cells were visualized by the co-expression of palmitoylated DsRed (DsRed-membrane: magenta) with GAS7b (cyan), to highlight the dense assembly that excludes the DsRed-membrane. Scale bars: $10 \mu \mathrm{m}$. b, c Time-lapse images of HeLa cells expressing GFP-GAS7b. Arrows indicate the appearance of the sheet-like GAS7b patches at ruffles (b) and the sheet-like GAS7b patches that transformed into holes as shown in the $z$ sections (c). Scale bars: $5 \mu \mathrm{m}$. d Time-lapse images of GFP-GAS7b (cyan) expressed in GAS7-knockout RAW264.7 macrophages incorporating the phagocytosis substrate, zymosan (magenta), captured at $93 \mathrm{~s}$ intervals. Arrows indicate the zymosan incorporation. The section at the line is also shown. Scale bar: $5 \mu \mathrm{m}$. e Localizations of endogenous GAS7 (cyan) and actin filaments (magenta) in RAW264.7 macrophages incorporating zymosan (DIC, arrows). Scale bar: $5 \mu \mathrm{m}$. f, $\mathbf{g} \mathrm{N}$-WASP (f) and Arp3 (g) with GFP-GAS7b (cyan) stably expressed at the endogenous GAS7 level in GAS7-knockout RAW264.7 macrophages incorporating zymosan (DIC, arrow). Scale bar: $5 \mu \mathrm{m}$. h Localizations of GFP-GAS7b, $\Delta$ FFL2, D207R and K209E mutants (cyan) in GAS7b-knockout RAW264.7 macrophages after the incorporation of zymosan (magenta). Scale bar: $5 \mu \mathrm{m}$. i, j Quantification of zymosan incorporation by GAS7b-knockout RAW264.7 macrophages stably expressing GFP, GFP-GAS7b and $\Delta$ FFL2 mutant (i) or GFP, GFP-GAS7b, GFPGAS7b, D207R mutant and K209E mutant (j). Dots represent zymosan incorporation by each cloned cell line. GFP alone was expressed as a control. $P$ values were determined by the two-tailed Student's $t$ test relative to GFP-expressing GAS7-knockout cells are shown. Error bars show SD. Source data are provided as a Source Data file. k, I Time courses of fluorescence recovery after photobleaching for GFP-GAS7 F-BAR and GFP-GAS7b on GUVs prepared from the PC, PE and PS lipids at a ratio of 20:20:60 (k), and GFP-GAS7 F-BAR and GFP-GAS7b expressed in HeLa cells and GFP-GAS7b expressed at the endogenous level in GAS7-knockout RAW264.7 macrophages (I). Source data are provided as a Source Data file. Error bars show SD 
a

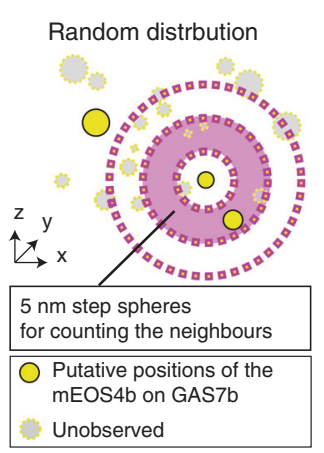

Simulated random GAS7b dimers

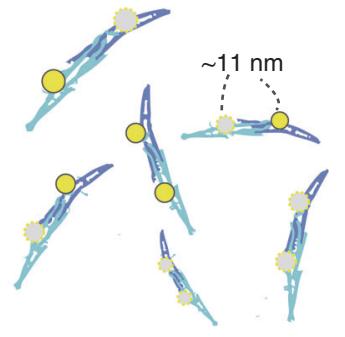

Simulated GAS7 FFO sheet

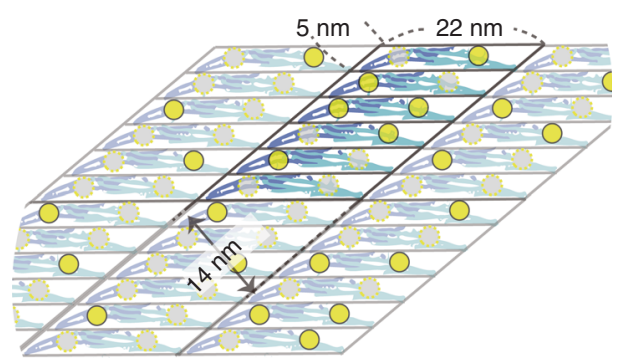

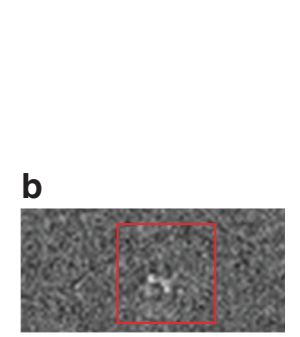

c
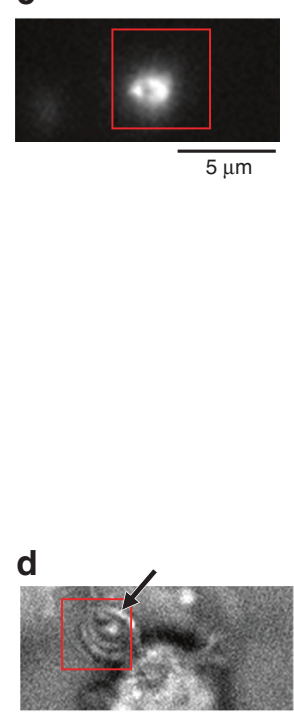

e

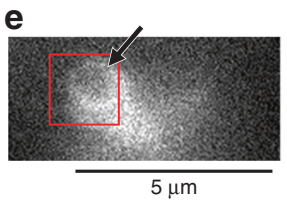

f
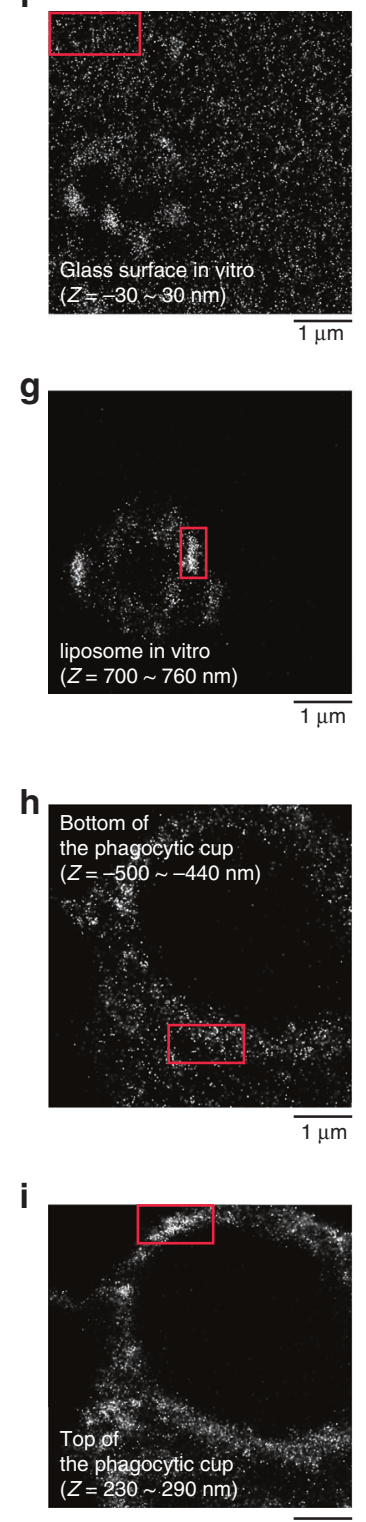

$\overline{1 \mu \mathrm{m}}$

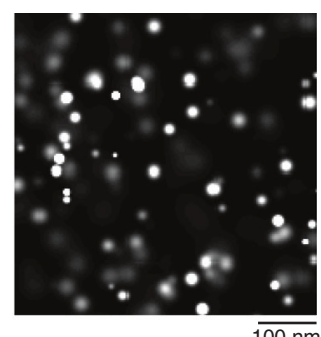

$\overline{100 \mathrm{~nm}}$

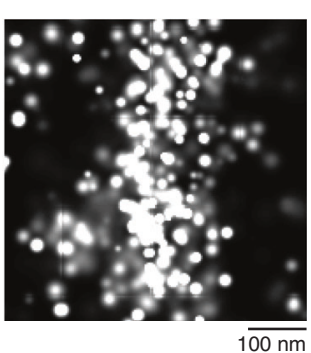

$\overline{100 \mathrm{~nm}}$

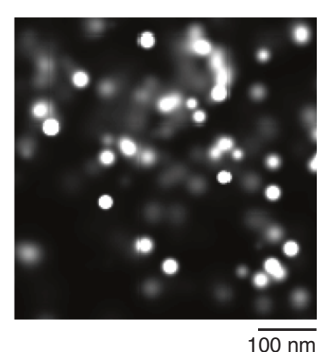

$\overline{100 \mathrm{~nm}}$

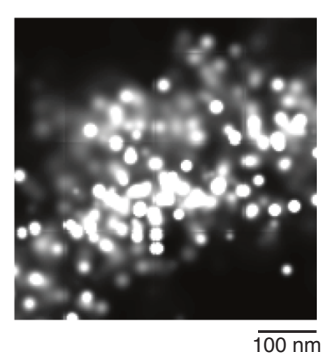

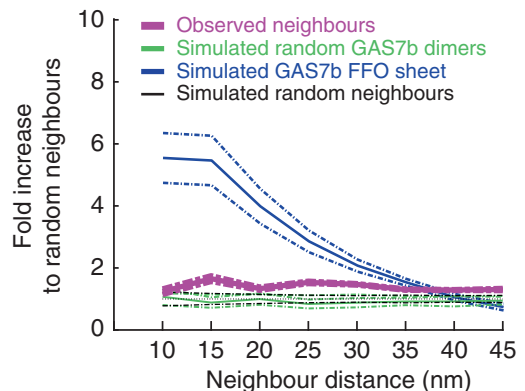
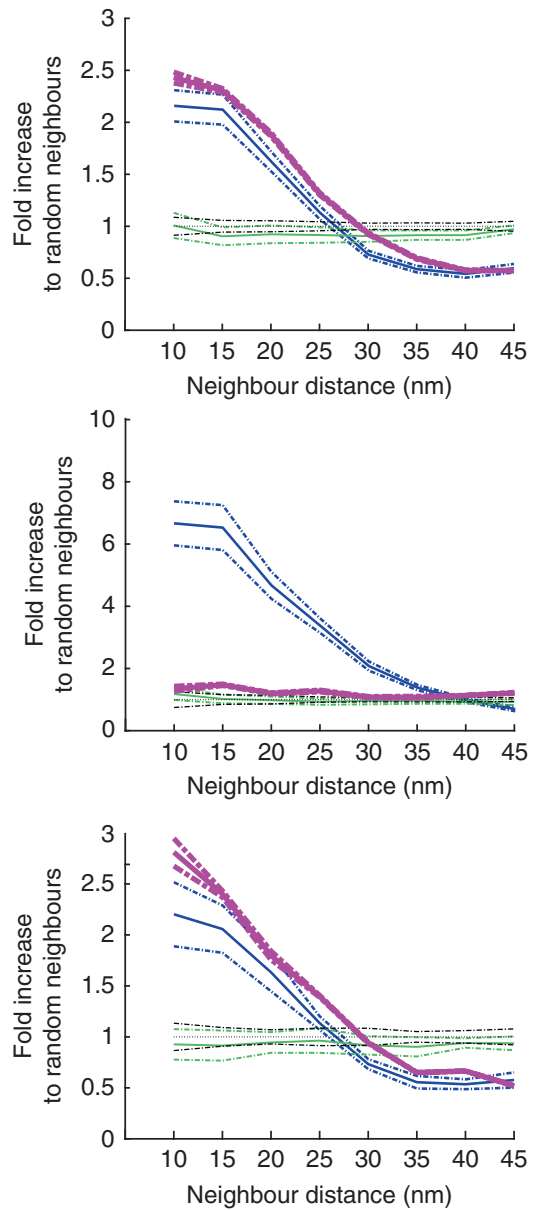

observed on liposomes and the tops of phagocytic cups had neighbours occurring with similar frequencies to those of the simulated FFO sheets (Fig. 4g, i). Interestingly, these GAS7b signals were sometimes aligned adjacent to each other (Fig. 4g, i). These data support the proposal that the GAS7 assembly into sheets occurs on both liposomes and phagocytic cups.

\section{Discussion}

Most BAR domain superfamily proteins are involved in the generation of membrane structures with submicron diameters, including clathrin-coated pits, caveolae, filopodia, endosomes and so on, through their membrane-binding abilities ${ }^{6,34}$. Several BAR superfamily proteins are involved in forming and binding to 
Fig. 4 GAS7b sheets on liposomes and phagocytic cups of macrophages. a Illustration of the possible spatial distributions of super-resolution signals. Randomly distributed signals, signals from randomly placed GAS7b dimers and signals from GAS7b forming FFO sheets are schematically illustrated. The alignment of the FFOs in the simulated sheet was considered to be lateral, as illustrated. Dashed magenta circles indicate the counting of the signals neighbouring a signal. b-e Phase-contrast (b, d) and epi-fluorescent (c, e) images of liposomes (b, c) and phagocytic cups (d, e) using mEOS4b-GAS7b, with arrows indicating incorporated zymosan. Scale bar: $5 \mu \mathrm{m}$. $\mathbf{f - i}$ Super-resolution images showing the single-molecule localization of photoconvertible mEOS4b-tagged GAS7b. Left panels are the $60 \mathrm{~nm}$ slices of mEOS4b signals on glass (f), on liposomes (g), at the bottom of a phagocytic cup (h), at the top of a phagocytic cup (i) and at the indicated observation depths of the regions marked by red rectangles in $\mathbf{b}$, $\mathbf{c}$ and $\mathbf{d}$, e. Middle panels are enlarged images of the parts enclosed in red rectangles in the left panels. Graphs on the right show the occurrences of observed neighbour signals (shown in magenta) dependent on the neighbour distances within the regions enclosed by red rectangles in the left panels. The same numbers of signals from the simulated randomly placed GAS7b dimers and the simulated FFO sheet, under the assumption of the 10\% observation of the total molecules, are shown in green and blue, respectively. The occurrence is described as the fold increase in the same numbers of the randomly distributed simulated signals. Dashed lines represent \pm SD of 20 trial simulations. Scale bars: $1 \mu \mathrm{m}$ (left) and $100 \mathrm{~nm}$ (middle)

relatively flat membranes, including lamellipodia, cell-cell junctions and membranes before their transformation into clathrin-coated pits. Lamellipodia contain the I-BAR domain protein IRSp $53^{35}$, as well as the F-BAR domain proteins CIP4 $4^{36}$, FBP $17^{37}$, srGAP1- $4^{38}$ and FCHSD $1 / 2 / \mathrm{Nwk}^{30}$. Cell-cell junctions contain the I-BAR domain protein PinkBAR ${ }^{24}$. The relatively flat membrane before endocytosis contains FCHSD $1 / 2^{39}$ and FCHol $/ 2^{40}$. However, the means by which the BAR superfamily proteins form twodimensional oligomers on these structures have remained enigmatic. Phagocytic cups are considered to be analogous structures to lamellipodia ${ }^{1,41-43}$. The studies of the BAR domain superfamily members involved in phagocytic cup formation have been limited to FBP17 in macrophages ${ }^{44}$, IBARa in dictyostelium ${ }^{45}$ and Bin2 in leucocytes ${ }^{46}$. None of these proteins has been suggested to form a two-dimensional oligomeric assembly in the phagocytic cup. Our data now indicate that the GAS7b sheet formation is critical in the membrane deformation during phagocytosis.

We showed that the N-surface is unlikely to be the membranebinding surface of the GAS7 F-BAR domain upon the oligomer formation. Instead, GAS7 F-BAR likely binds to the membrane through the surface close to the side surface. The side surface was previously shown to be used by the F-BAR domain of FBP17 to bind to the flat membrane, presumably before it deformed the membrane into a tubular shape by binding through the concave $\mathrm{N}$-surface ${ }^{10}$. GAS7 did not induce significant membrane deformation when it bound to flat membranes under our conditions. This might arise from the membrane binding preferentially on the side surface of the F-BAR domain, as determined by the mutagenesis analysis (Fig. 3, Supplementary Fig. 5a). Although GAS7 alone did not induce prominent membrane deformation of the already micron-sized GUVs, GAS7 F-BAR in HeLa cells was localized at micron-sized, cup-like assemblies (Supplementary Fig. 5a). Therefore, the GAS7 F-BAR domain itself might participate in micron-sized relatively flat membrane remodelling, under as yet unidentified conditions.

Most membrane structures involving BAR superfamily proteins are accompanied by Arp $2 / 3$ complex-mediated actin polymerization. The $\mathrm{SH} 3$ domains in the BAR superfamily proteins bind to the WASP family proteins, including N-WASP and WAVE, which then activate the Arp2/3 complex. GAS7b binds to N-WASP with the WW domain, and GAS7cb has the $\mathrm{SH} 3$ domains in addition to the WW domain ${ }^{13,14}$. These domains bind to N-WASP, thus regulating the Arp2/3 complex-mediated actin polymerization ${ }^{47-49}$. The FBAR domain alone failed to rescue phagocytosis, suggesting the requirement of the WW domain in GAS7b to support phagocytosis. The membrane binding of GAS7 was also essential, as the proteins with mutations in the F-BAR domain, which inhibited membrane binding, also failed to restore phagocytosis. Therefore, the WASP family protein-mediated actin dynamics regulation may cooperate with GAS7 for phagocytic cup formation. The formation of phagocytic cups is considered to share mechanisms with lamellipodia,
Arp2/3-dependent protrusive structures for cell migration, in which the Arp $2 / 3$ complex regulators including N-WASP play a significant role. The Arp2/3-complex-mediated actin polymerization is also necessary for phagocytic cup formation ${ }^{50}$. Interestingly, GAS7knockout cells exhibited defects in lamellipodia formation, indicating that GAS7 is part of the shared mechanisms between phagocytosis and lamellipodia formation, including actin dynamics regulation ${ }^{41,43}$.

The BAR domain superfamily proteins assemble on the membrane and activate N-WASP-mediated actin polymerization ${ }^{47-49}$. The high-density assembly of the BAR superfamily proteins on the membrane results in the accumulation of other domains, including the WW and $\mathrm{SH} 3$ domains, to near milli-molar concentrations. The electron microscopic and super-resolution analyses support such a high concentration of GAS7 on the membrane. The actin polymerization by the Arp $2 / 3$ complex is enhanced by the highly concentrated N-WASP-binding domains on the membranes ${ }^{49,51}$. Further analyses will clarify how GAS7 sheet formation and actin polymerization cooperatively regulate membrane deformation during phagocytosis.

\section{Methods}

Gene cloning, protein purification and crystallization. Mouse GAS7cb (GenBank accession XM_006532202.1) was cloned from a mouse brain complementary DNA library by PCR. The primers used in this study were listed in Supplementary Table 2. The DNA sequences encoding the F-BAR domains of mouse GAS7cb (166-476 aa) and GAS7cb (1-476 aa) were cloned into the pCold II vector (Takara Bio, Japan), using the Bam HI and SalI restriction sites to include an N-terminal affinity tag $\left(\mathrm{His}_{6}\right)$. To express the selenomethionine (SeMet)-substituted protein, Escherichia coli (E. coli) strain JM109 (Takara Bio) was transformed with the expression plasmid for the F-BAR domain. Escherichia coli cells were grown in M9 minimal medium at $37^{\circ} \mathrm{C}$. When the $\mathrm{OD}_{600}$ reached 0.7 , a final concentration of $60 \mathrm{mg} \mathrm{l}^{-1} \mathrm{~L}$-SeMet; $100 \mathrm{mg} \mathrm{l}^{-1}$ each of L-threonine, L-lysine hydrochloride and L-phenylalanine; and $50 \mathrm{mg} \mathrm{l}^{-1}$ each of L-leucine, L-isoleucine and L-valine were added to prevent methionine production. The protein was overexpressed at $15^{\circ} \mathrm{C}$ in the presence of $0.5 \mathrm{mM}$ isopropyl- $\beta$-D-1-thiogalactopyranoside. The harvested $E$. coli cells were resuspended in buffer containing $20 \mathrm{mM}$ Tris- $\mathrm{HCl}$ (pH 7.5), $0.50 \mathrm{M}$ $\mathrm{NaCl}, 0.50 \mathrm{M} \mathrm{MgCl}_{2}, 10 \mathrm{mM}$ 2-mercaptoethanol and $1 \mathrm{mM}$ phenylmethanesulfonyl fluoride (PMSF), and were disrupted with an ultrasonic homogenizer. The lysate was centrifuged, and the supernatant was loaded on a Ni-Sepharose 6 Fast Flow column (GE Healthcare). The column was washed with $20 \mathrm{mM}$ Tris- $\mathrm{HCl}$ (pH 7.5) buffer, containing $0.80 \mathrm{M} \mathrm{NaCl}, 10 \mathrm{mM}$ 2-mercaptoethanol and $20 \mathrm{mM}$ imidazole. The protein was eluted with $20 \mathrm{mM}$ Tris- $\mathrm{HCl}$ ( $\mathrm{pH} 8.5$ ) buffer, containing $0.80 \mathrm{M}$ $\mathrm{NaCl}$ and $0.30 \mathrm{M}$ imidazole. The eluted protein was further purified on a HiLoad Superdex 200 pg 16/600 column (GE Healthcare), using $20 \mathrm{mM}$ Tris- $\mathrm{HCl}$ (pH 7.5) buffer containing $0.20 \mathrm{M} \mathrm{NaCl}$ and $10 \mathrm{mM}$ 2-mercaptoethanol, and was concentrated with an Amicon Ultra filter (Merck Millipore). The crystals of the F-BAR domain (13.3 $\mathrm{mg} \mathrm{ml}^{-1}$ ) were grown using the vapour-diffusion method, by 1:1 mixing of the protein solution with a reservoir composed of $12 \%$ PEG $4000,4 \%$ PEG 8000, 0.16 M ammonium sulphate, $80 \mathrm{mM}$ HEPES-NaOH (pH 7.5) and $20 \mathrm{mM}$ MES (pH 6.5).

The E. coli strain Rosetta 2 was transformed with the expression plasmid for $\mathrm{GAS} 7 \mathrm{cb}$, and the protein was overexpressed in $\mathrm{LB}$ medium at $15^{\circ} \mathrm{C}$. The bacterial pellet was resuspended in buffer containing $20 \mathrm{mM}$ Tris- $\mathrm{HCl}(\mathrm{pH} 7.5), 0.80 \mathrm{M}$ $\mathrm{NaCl}, 10 \%$ glycerol, $1 \mathrm{mM}$ DTT and $1 \mathrm{mM}$ pefablock or PMSF, and the bacteria were disrupted with an ultrasonic homogenizer. The lysate was centrifuged, and the supernatant was mixed with $\mathrm{Ni}$-Sepharose and washed once in the batch mode. 
The beads were then transferred to a column and washed with $150 \mathrm{ml}$ of $20 \mathrm{mM}$ Tris- $\mathrm{HCl}$, pH 7.5, containing $0.80 \mathrm{M} \mathrm{NaCl}, 0.2 \mathrm{mM}$ pefablock or PMSF, $1 \mathrm{mM}$ dithiothreitol (DTT) and $20 \mathrm{mM}$ imidazole. The protein was eluted with step gradients of 40,60, 80 and $500 \mathrm{mM}$ imidazole in $20 \mathrm{mM}$ Tris- $\mathrm{HCl}$ (pH 7.5) buffer, containing $0.8 \mathrm{M} \mathrm{NaCl}, 0.2 \mathrm{mM}$ pefablock or PMSF and $1 \mathrm{mM} \mathrm{DTT}$. The GAS7cb fractions were further purified on a HiLoad Superdex $200 \mathrm{pg}$ 16/600 column (GE Healthcare), using $20 \mathrm{mM}$ Tris- $\mathrm{HCl}$ (pH 7.5) buffer containing $0.3 \mathrm{M} \mathrm{NaCl}$ and $1 \mathrm{mM}$ DTT. The protein $\left(15.0 \mathrm{mg} \mathrm{ml}^{-1}\right)$ was concentrated with an Amicon Ultra filter and then crystallized using the vapour-diffusion method, by 1:1 mixing with the crystal reservoir composed of $0.1 \mathrm{M}$ sodium citrate, $\mathrm{pH} 5.0,1.2 \mathrm{M}$ sodium formate, $0.2 \mathrm{M} \mathrm{NaCl}$ and $1-4 \%$ ethylene glycol.

To express GAS7b, E. coli strain JM109 or BL21 was transformed with the pGEX6P1 plasmid, containing the mouse GAS7b (aa 62-476; GAS7cb amino-acid residue numbers) gene cloned into the BamHI site. After immobilization of the protein on the Glutathione Sepharose (GE Healthcare) and washing, the GST tag was cleaved by Precision protease, and the cleaved proteins were collected in $10 \mathrm{mM}$ Tris- $\mathrm{HCl}$ (pH 7.5) buffer, containing $150 \mathrm{mM} \mathrm{NaCl}$ and $1 \mathrm{mM} \mathrm{EDTA}^{52}$

To express the F-BAR domain of GAS7 for light and electron microscopy, $E$. coli strain JM109 or BL21 was transformed with the pGEX6P1 plasmid, containing mouse GAS7cb (166-476 aa) cloned into the BamHI site. The purification was performed as for GAS7b. For electron microscopy, GAS7b, the F-BAR domain of GAS7 and GAS7cb were purified by gel filtration, in a similar manner to the proteins used for crystallization.

Data collection and structure determination. Crystals of the F-BAR domain or GAS7cb were soaked in a cryo-protective solution, composed of 22\% PEG 4000, $70 \mathrm{mM}$ ammonium sulphate, $80 \mathrm{mM}$ HEPES- $\mathrm{NaOH}$ (pH 7.5) and $15.5 \%$ ethylene glycol or $25 \%$ ethylene glycol, respectively, and flash cooled in a nitrogen cryostream. Data sets were collected at the Photon Factory beamline BL-1A (Tsukuba, Japan) and the SPring-8 beamlines BL32XU, BL38B1 and BL44XU (Hyogo, Japan), and were processed with $\mathrm{XDS}^{53}$ or HKL2000 ${ }^{54}$.

The structure of the GAS7 F-BAR domain was solved by the single-wavelength anomalous dispersion method, using the program autoSHARP ${ }^{55}$. The structure of GAS7cb was solved by the molecular replacement method, using the structure of the GAS7 F-BAR domain as the search model, with PHENIX ${ }^{56}$. Refinement was performed with PHENIX. Figures were created with the program PyMol (http://www.pymol.org). The Ramachandran plot analysis indicated that $95.6 \%$ (GAS7 F-BAR domain) and 78\% (GAS7cb) of the residues were in the favoured regions and $1.5 \%$ (GAS7 F-BAR domain) and 3.5\% (GAS7cb) of the residues were in the outlier regions.

MD simulations. A 1- $\mu \mathrm{s} \mathrm{MD}$ simulation of F-BAR in solution ( $150 \mathrm{mM} \mathrm{NaCl}$ ) was performed using Gromacs 2018.1 $1^{57,58}$. The system was brought to thermodynamic equilibrium at $300 \mathrm{~K}$ and $1 \mathrm{~atm}$, using the Nosé-Hoover thermostat and the Parrinello-Rahman barostat. The equations of motion were integrated with a time step of $2 \mathrm{fs}$. The long-range Coulomb energy was evaluated using the particle mesh Ewald method. The CHARMM $36 \mathrm{~m}$ force field ${ }^{59}$ was used.

Fluorescence microscopy of liposomes. GUVs were prepared by natural swelling. The lipids, phosphatidylcholine (PC) (P3841, Sigma-Aldrich), phosphatidylethanolamine (PE) (P7693, Sigma-Aldrich), PS (P5660, Sigma-Aldrich) and PIP 3 (P3916, Echelon Biosciences), were prepared in chloroform in a glass tube at the indicated molar ratios, to a final total concentration of $0.1 \mathrm{mM}$ lipids, dried under nitrogen gas and subsequently incubated under vacuum. Subsequently, $20 \mu \mathrm{l}$ of buffer containing $10 \mathrm{mM}$ Tris- $\mathrm{HCl}$ (pH 7.5), $300 \mathrm{mM}$ sucrose and $1.0 \mathrm{mM}$ EDTA was added to the glass tube, which was then sealed and incubated at $45^{\circ} \mathrm{C}$ for $8 \mathrm{~min}$ (prehydration). A $230 \mu \mathrm{l}$ aliquot of the same buffer was added, and the tube was resealed and incubated at $37^{\circ} \mathrm{C}$ for $2 \mathrm{~h}$ to produce the GUV suspension. After this incubation, the GUV suspension was mixed with buffer containing $10 \mathrm{mM}$ Tris$\mathrm{HCl}$ (pH 7.5), $1 \mathrm{mM}$ EDTA and $150 \mathrm{mM} \mathrm{NaCl}$ in a 1:1 ratio, and then GFP-GAS7b and GUVs were incubated for $5 \mathrm{~min}$ at room temperature. The mixture was combined with $1 \%$ bovine serum albumin (BSA) as a blocking reagent, and observed with an FV1000D (Olympus) confocal microscope.

Electron microscopy. Lipid monolayers containing PC, PE and PS at a molar ratio of 1:1:3 were formed on the carbon-coated EM grid in buffer containing $50 \mathrm{mM}$ Tris- $\mathrm{HCl}(\mathrm{pH} 8.0)$ and $100 \mathrm{mM} \mathrm{NaCl}$, and then covered with proteins $\mathrm{s}^{21,22}$. The $\mathrm{EM}$ grids were then stained with uranyl acetate.

Liposome co-sedimentation assays. The in vitro liposome-binding analysis 23,60 was performed using the GAS7 in Fig. 2f, j, l, m or the GFP-GAS7 in Fig. 2g-i, k. Briefly, liposomes were made from the Folch fraction, a brain total lipid fraction rich in PS (Sigma-Aldrich, B1502). Liposomes were also made by mixing PC (Sigma-Aldrich, P3841), PE (Sigma-Aldrich, P7693), PS (Sigma-Aldrich P5660) and $\mathrm{PIP}_{3}$ (P-3916, Echelon Biosciences). Lipids in chloroform were dried under nitrogen gas and subsequently incubated under vacuum. The dried lipids were resuspended in buffer containing $10 \mathrm{mM}$ Tris- $\mathrm{HCl}(\mathrm{pH}$ 7.5), $1 \mathrm{mM}$ EDTA and $200 \mathrm{mM} \mathrm{NaCl}$ for Fig. $2 \mathrm{~g}$, k, in high-salt buffer containing $10 \mathrm{mM}$ Tris- $\mathrm{HCl}$ (pH 7.5), $1 \mathrm{mM}$ EDTA and $300 \mathrm{mM} \mathrm{NaCl}$ for Fig. $2 \mathrm{~h}$, and buffer containing $10 \mathrm{mM}$
Tris- $\mathrm{HCl}$ (pH 7.5), $1 \mathrm{mM}$ EDTA and $150 \mathrm{mM} \mathrm{NaCl}$ for Fig. $2 \mathrm{f}, \mathrm{i}, \mathrm{j}, \mathrm{l}$, and then incubated for $1 \mathrm{~h}$ at $37^{\circ} \mathrm{C}$ to form liposomes. Subsequently, $0.5 \mu \mathrm{M}$ protein and $0.2 \mathrm{mg} \mathrm{ml}^{-1}$ (Fig. 2f, l) or $0.4 \mathrm{mg} \mathrm{ml}^{-1}$ liposomes (Fig. $2 \mathrm{~g}-\mathrm{k}$ ) were incubated in the same respective buffers for $20 \mathrm{~min}$ at room temperature. The liposomes were then precipitated by centrifugation at 50,000 r.p.m. for $20 \mathrm{~min}$ in a TLA100 rotor (Beckman Coulter). The pellet and supernatant were fractionated by sodium dodecyl sulfate-polyacrylamide gel electrophoresis (SDS-PAGE) and visualized by staining with Coomassie Brilliant Blue.

Crosslinking assays. The BS(PEG)5 (PEGylated bis(sulfosuccinimidyl)suberate) linker, with spacer arm length of $21.4 \AA$, was used at the indicated concentrations. The proteins $(0.5 \mu \mathrm{M})$, in buffer containing $10 \mathrm{mM}$ HEPES (pH 8.0$), 150 \mathrm{mM} \mathrm{NaCl}$ and $1 \mathrm{mM}$ EDTA, were incubated at room temperature for $20 \mathrm{~min}$ at $37^{\circ} \mathrm{C}$ with or without liposomes. Then, the proteins were mixed with $\mathrm{BS}(\mathrm{PEG}) 5$ and were incubated for $10 \mathrm{~min}$ in $37^{\circ} \mathrm{C}$. The reaction was quenched by adding Tris- $\mathrm{HCl}(\mathrm{pH}$ 7.5) to a final concentration of $25 \mathrm{mM}$, incubated for $15 \mathrm{~min}$ at room temperature and then analysed by SDS-PAGE and western blotting.

Cell culture. GAS7, $\triangle$ FFL1 (aa 171-197 deletion, GAS7cb residue numbers), $\triangle$ FFL2 (aa 209-216 deletion) and all of the amino-acid substitution mutants (all described in terms of the GAS7cb amino-acid residue number) were cloned into the pEGFP-C1 vector, and EGFP was substituted with its brighter variant, Venus ${ }^{61}$ The DsRed membrane was expressed using the pDsRed-monomer-Mem vector (Clontech). HeLa cells were cultured in Dulbecco's modified Eagle's medium (DMEM) supplemented with $10 \%$ fetal bovine serum (FBS) ${ }^{52}$. Transfection was performed with Lipofectamine LTX and PLUS reagents (Invitrogen), according to the manufacturer's instructions. Cells were observed with a confocal microscope (Olympus FV1000D).

RAW264.7 cells were cultured in DMEM supplemented with 10\% FBS. Transfection of RAW264.7 cells $\left(1 \times 10^{5}\right.$ cells per $\left.10 \mu \mathrm{l}\right)$ with siRNA $(1 \mu \mathrm{l}, 20 \mu \mathrm{M})$ was performed via electroporation with 1 pulse from a NEON transfection system (Invitrogen), at $1680 \mathrm{~V}$ and $20 \mathrm{~ms}$. The GAS7 siRNA solution was a mixture of three siRNAs from Invitrogen (GAS7HSS144787, GAS7HSS144788 and GAS7HSS144789). The control siRNA was also from Invitrogen.

Knockout and retrovirus-mediated gene transfer. The CRISPR/Cas9 system was used as described previously ${ }^{62}$. The guide RNA targeting the first exon of GAS7b (GGCGGAGGGGGGACCATTCC) was designed using the server http://crispr.mit. $e^{e d u^{63}}$ and inserted into the pX330 vector ${ }^{62}$. After transfection, the cells were cloned by monitoring the GFP fluorescence from the reporter plasmid pCAG-EGxxFP with the GAS7 genome fragment, using a fluorescence-activated cell sorter (FACSAria (BD)). GFP, GFP-GAS7, mEOS4b-GAS7b and GAS7 mutants were each introduced into the pMXs vector and expressed in GAS7-knockout cells, using a retrovirus produced by PLAT-A packaging cells ${ }^{64}$. The cells were then cloned and isolated using a cell sorter. Clones with GAS7 expression similar to that of the parental cells were selected, and examined for their phagocytic activity.

Phagocytosis assays. Red-coloured zymosan (Invitrogen) or zymosan (Sigma) was opsonized with bovine serum albumin and incubated at $0.2 \mathrm{mg} \mathrm{ml}^{-1}$ with cells at $37^{\circ} \mathrm{C}$. The cells were either subjected to live-cell imaging or fixed after $1 \mathrm{~h}$. Live images were obtained with a confocal microscope (Olympus FV1000D) at $37^{\circ} \mathrm{C}$ in a $5 \% \mathrm{CO}_{2}$ atmosphere.

Wild-type or GAS7-knockout RAW264.7 cells grown on cover glasses were incubated with red-coloured zymosan (Invitrogen) or red fluorescent latex beads $\left(2 \mu \mathrm{m}\right.$, Sigma) coated with purified rabbit IgG (Sigma), for $1 \mathrm{~h}$ at $37^{\circ} \mathrm{C}$. The cells were washed, fixed with $4 \%$ paraformaldehyde for 20 min and mounted. Approximately 100-200 cells from six to seven randomly selected fields were examined, to determine the number of incorporated particles.

Immunofluorescent staining. Cells grown on cover glasses were treated with BSAcoated zymosan beads for $30 \mathrm{~min}$, and then fixed with $4 \%$ paraformaldehyde in the appropriate medium at room temperature for $20 \mathrm{~min}$. The cells were permeabilized with $0.5 \%$ Triton X-100 in Tris-buffered saline (TBS) at room temperature for 20 min. The samples were blocked with $3 \%$ BSA and $10 \%$ goat serum in TBS containing $0.1 \%$ Triton X-100 (TBS-T) for $1 \mathrm{~h}$, and then incubated with primary antibodies in Can Get Signal immunostain solution (TOYOBO) for $2 \mathrm{~h}$. After washing with TBS-T, the cells were incubated with fluorescently labelled secondary antibodies and phalloidin for $1 \mathrm{~h}$. After washing, the cells were mounted using Prolong Gold (Thermo Fisher/Invitrogen).

The following antibodies were used: mouse anti-GAS7 (clone 2F6, Origene, TA501756, 1:100 dilution); mouse anti-integrin- $\alpha \mathrm{M}$ (clone Ox42, Santa Cruz Biotechnology, sc-53086, 1:100) and anti-CD206 (Santa Cruz Biotechnology, sc376108, 1:100); rabbit anti-N-WASP (clone 30D10, Cell Signaling, 4848, 1:50); mouse anti-Arp3 (clone FMS338, Abcam, ab49671, 1:250); rabbit anti-GFP (MBL, 598); rat anti-GFP (clone GF090R, Nacalai, 040080, 1:1000); Alexa Fluorconjugated highly cross-adsorbed anti-mouse or anti-rabbit antibodies (Thermo Fisher, A-11034, A-11036, A-21245, A-11029, A-11031, A-21236, 1:400) and Alexa Fluor-conjugated phalloidin (Thermo Fisher/Invitrogen, A12380, 1:100); and CF488A-labelled anti-rat IgG antibody (Biotium, 20023, 1:400). 
Images of cells were obtained using an FV1000 laser-scanning confocal microscope (Olympus) equipped with a $\times 100 \mathrm{NA} 1.45$ oil lens (Olympus) at room temperature.

Western blotting. After SDS-PAGE, the proteins in the gel were transferred onto the membrane (Immobilon P, IPVH00010, Merck Millipore) by using Trans-Blot SD Semi-Dry Transfer Cell (Bio-Rad). The membrane was blocked with 5\% skim milk in PBS supplemented with $0.05 \%$ Tween-20 (PBS-T). Then, the proteins were examined by mouse anti-GAS7 (Origene, 2F6, TA501756), anti-actin (Merck Millipore, MAB1501), anti-GFP (MBL, 598) and anti-glyceraldehyde-3-phosphate dehydrogenase (Santa Cruz Biotechnology, sc-166574) antibodies as primary antibody at 1:1000 dilution, followed by the secondary antibody of anti-mouse or rabbit IgG alkaline phosphatase conjugate (Promega) in PBS-T with 1:10000 dilution. The proteins were detected by 5 -bromo-4-chloro-3-indoryl phosphate/ nitroblue tetrazolium (Roche).

FRAP and time-lapse imaging. Cells on coverslips were placed in the microscope's (FV1000, Olympus) humidified chamber with $5 \% \mathrm{CO}_{2}$. The region of interest was bleached by a $473 \mathrm{~nm}$ laser for $1 \mathrm{~s}$. The fluorescence intensities were analysed by the Image J (NIH) software. The fluorescence intensity before bleaching was set to $100 \%$. Fluorescence after bleaching was considered to be $0 \%$.

For the analysis of lamellipodia, cells were transfected with pCAH-LifeactEGFP $^{65}$ using Lipofectamine 3000 (Invitrogen), cultured for 1 day and then cultured in glass-bottom dishes (IWAKI) for 1 day. Time-lapse imaging was performed using an FV1000 (Olympus) laser-scanning confocal microscope at $30 \mathrm{~s}$ intervals.

Super-resolution microscopy observation. The photoconvertible fluorophore, mEOS $4 \mathrm{~b}$, was used because it can be converted to a red fluorescent protein by ultraviolet (UV) irradiation, even after fixation ${ }^{33}$. For the observation of mEOS4bGAS7b on liposomes, $50 \mu \mathrm{l}$ portions of liposomes, composed of $0.125 \mathrm{mg} \mathrm{ml}^{-1}$ lipids with $1 \%$ of biotin-PE (Avanti) in $300 \mathrm{mM}$ sucrose, were placed on glassbottom dishes coated with streptavidin and biotin-BSA for immobilization, as described previously ${ }^{37}$. The liposomes were overlaid with $10 \mu \mathrm{l}$ of $4 \mu \mathrm{M}$ mEOS4bGAS7b in $10 \mathrm{mM}$ Tris buffer (pH 7.5), containing $150 \mathrm{mM} \mathrm{NaCl}$ and $1 \mathrm{mM}$ EDTA, and sandwiched with a cover glass. After $15 \mathrm{~min}$ at room temperature, the liposomes were fixed with $4 \%$ paraformaldehyde and $0.2 \%$ glutaraldehyde in HEPESbuffered saline for $20 \mathrm{~min}$

GAS7-knockout RAW264.7 cells expressing mEOS4b-GAS7b were cultured on glass coverslips and incubated with zymosan for $1 \mathrm{~h}$. The cells were then fixed in $4 \%$ paraformaldehyde with $0.2 \%$ glutaraldehyde (electron microscopy grade) in HEPES-buffered saline, containing $30 \mathrm{mM}$ HEPES (pH 7.5), $100 \mathrm{mM} \mathrm{NaCl}$ and $2 \mathrm{mM} \mathrm{CaCl}$ for $20 \mathrm{~min}$.

The fixed samples were reduced with PBS $\left(0.1 \% \mathrm{NaBH}_{4}\right)$ for $5 \mathrm{~min}$. The samples were then stored in PBS with $1 \%$ polyvinyl alcohol and $10 \mathrm{mM}$ cysteamine (MEA). The samples were sealed with a 1:1:1 vaseline lanolin paraffin mixture or vaseline and then imaged using the N-STORM setup with an iXon DU-897E electronmultiplying charge-coupled device camera (Andor). The images were recorded with the NIS-elements software version 4.60 .00 and N-STORM version 4.0.0.215, using the 3 D-STORM mode. Images $\left(10 \times 10^{5}\right)$ were acquired continuously with a $12 \mathrm{~mW} 543 \mathrm{~nm}$ laser, with continuous activation by a $405 \mathrm{~nm}$ laser. mEOS4b emits stochastic signals as it is converted from green to red by UV or violet light activation $^{66-68}$. Red signals were erased upon observation by the $543 \mathrm{~nm}$ laser

Super-resolution microscopy data analysis. Each single-molecule localization was analysed using the NIS-elements software provided by Nikon. Signals in the same pixel ( $160 \mathrm{~nm}$ square) in continuous images were considered to be derived from the same molecule and then merged. The $x y$ drifts between $z$ stacks were collected, using either fiducial markers or autocorrelation between images. The coordinates of the signals were exported and drift corrected using ThunderSTORM ${ }^{69}$.

The visualization of molecules was performed using the MATLAB software (Mathworks). Molecule localization was shown by the probabilities of the mixed Gaussian distribution of the signals, where the accuracy of each signal was used as the deviation (sigma) for the mixed Gaussian distribution. The deviation in the depth direction $(z)$ was set to be twice that in the focal plane $(x y)$.

The randomness of the signals was analysed using MATLAB with a modified Ripley's $k$ function analysis ${ }^{70}$. The region of interest was selected, and the distances of the observed signals to their neighbours were calculated using the threedimensional coordinates of the signals. The frequency of the neighbours in $5 \mathrm{~nm}$ step distances was calculated (i.e., the numbers of neighbours from $5 \times i$ to $5(i+1)$ $\mathrm{nm}$ distances from a signal were counted, $i=2-9$ ). The same number of randomly placed signals was generated as simulations, which were used for determining the fold increase in the observed neighbours as compared to the neighbours of random signals. The random signals were simulated 20 times, and these frequencies of neighbours were used to estimate the fold increases in the number of observed neighbours, as shown with \pm SD. Frequencies below $10 \mathrm{~nm}$ distances were not shown because of the small number of signals, which result in a large variance in the fold increase.
The simulation for the comparison to the observed super-resolution signals was performed using the same number of signals as that of the observed signals to be compared. The simulated dimers were generated by pairs of labels with a distance of $11 \mathrm{~nm}$. The simulated FFOs were the string of dimers with a spacing of $5 \mathrm{~nm}$ and a $40^{\circ}$ tilt, as in the FFOs in the crystal. To generate the simulated FFO sheets, the FFOs were aligned in parallel, but the anti-parallel alignments gave almost identical results in the comparison. In total, $40 \%$ of the molecules were considered to form sheets, whereas the rest of the molecules were random dimers. We estimated the averaged concentration of the proteins and the number of proteins in the cells by western blotting using the purified GAS7b as the standard, and these values were compared with the total number of signals in RAW264.7 cells. Consequently, 10\% of the labels were assumed to be observed. Each signal had an accuracy of $20 \mathrm{~nm}$ in the simulation.

Statistical analyses. All data are expressed as the mean \pm SD, as indicated in the legends. Data for each condition were obtained from at least three independent experiments. Statistical analyses were performed using Microsoft Excel and Student's $t$ test. A value of $P<0.05$ was considered significant.

Reporting summary. Further information on research design is available in the Nature Research Reporting Summary linked to this article.

\section{Data availability}

Atomic coordinates and structure factors for the crystal structures of the GAS7 F-BAR domain and GAS7cb have been deposited in the Protein Data Bank, under the accession codes $6 \mathrm{IKN}$ and $6 \mathrm{IKO}$, respectively. The source data underlying Figs. $2 \mathrm{f}-\mathrm{m}, 3 \mathrm{i}-\mathrm{l}$ and Supplementary Figs. 1b-c, 4a-h, 6, 7d-f, h and i are provided as a Source Data File. Other data are available from the corresponding authors on reasonable request.

\section{Code availability}

The MATLAB codes and the source coordinates for Fig. 4 are available at GitHub .

Received: 21 November 2018; Accepted: 26 September 2019; Published online: 18 October 2019

\section{References}

1. Aderem, A. \& Underhill, D. M. Mechanisms of phagocytosis in macrophages Annu. Rev. Immunol. 17, 593-623 (1999).

2. Desjardins, M. ER-mediated phagocytosis: a new membrane for new functions. Nat. Rev. Immunol. 3, 280-291 (2003).

3. Gordon, S. Phagocytosis: an immunobiologic process. Immunity 44, 463-475 (2016).

4. Simunovic, M., Voth, G. A., Callan-Jones, A. \& Bassereau, P. When physics takes over: BAR proteins and membrane curvature. Trends Cell Biol. 25, 780-792 (2015)

5. Takenawa, T. \& Suetsugu, S. The WASP-WAVE protein network: connecting the membrane to the cytoskeleton. Nat. Rev. Mol. Cell. Biol. 8, 37-48 (2007)

6. Suetsugu, S., Kurisu, S. \& Takenawa, T. Dynamic shaping of cellular membranes by phospholipids and membrane-deforming proteins. Physiol. Rev. 94, 1219-1248 (2014).

7. Daumke, O., Roux, A. \& Haucke, V. BAR domain scaffolds in dynaminmediated membrane fission. Cell 156, 882-892 (2014)

8. Doherty, G. J. \& McMahon, H. T. Mechanisms of endocytosis. Annu. Rev Biochem. 78, 857-902 (2009).

9. Scita, G., Confalonieri, S., Lappalainen, P. \& Suetsugu, S. IRSp53: crossing the road of membrane and actin dynamics in the formation of membrane protrusions. Trends Cell Biol. 18, 52-60 (2008).

10. Frost, A. et al. Structural basis of membrane invagination by F-BAR domains. Cell 132, 807-817 (2008).

11. Frost, A., Unger, V. M. \& De Camilli, P. The BAR domain superfamily: membrane-molding macromolecules. Cell 137, 191-196 (2009).

12. Saarikangas, J. et al. Molecular mechanisms of membrane deformation by IBAR domain proteins. Curr. Biol. 19, 95-107 (2009).

13. You, J. J. \& Lin-Chao, S. Gas7 functions with N-WASP to regulate the neurite outgrowth of hippocampal neurons. J. Biol. Chem. 285, 11652-11666 (2010).

14. Tseng, R. C. et al. Growth-arrest-specific 7C protein inhibits tumor metastasis via the N-WASP/FAK/F-actin and hnRNP U/beta-TrCP/beta-catenin pathways in lung cancer. Oncotarget 6, 44207-44221 (2015)

15. Chang, P. Y., Kuo, J. T., Lin-Chao, S. \& Chao, C. C. Identification of rat Gas7 isoforms differentially expressed in brain and regulated following kainateinduced neuronal injury. J. Neurosci. Res. 79, 788-797 (2005).

16. Su, A. I. et al. A gene atlas of the mouse and human protein-encoding transcriptomes. Proc. Natl. Acad. Sci. USA 101, 6062-6067 (2004). 
17. Wu, C., Jin, X., Tsueng, G., Afrasiabi, C. \& Su, A. I. BioGPS: building your own mash-up of gene annotations and expression profiles. Nucleic Acids Res. 44, D313-D316 (2016).

18. Ju, Y. T. et al. Gas7: A gene expressed preferentially in growth-arrested fibroblasts and terminally differentiated Purkinje neurons affects neurite formation. Proc. Natl. Acad. Sci. USA 95, 11423-11428 (1998).

19. Okamura, Y. et al. COXPRESdb in 2015: coexpression database for animal species by DNA-microarray and RNAseq-based expression data with multiple quality assessment systems. Nucleic Acids Res. 43, D82-D86 (2015).

20. Araki, N., Johnson, M. T. \& Swanson, J. A. A role for phosphoinositide 3kinase in the completion of macropinocytosis and phagocytosis by macrophages. J. Cell Biol. 135, 1249-1260 (1996).

21. Ford, M. G. et al. Curvature of clathrin-coated pits driven by epsin. Nature 419, 361-366 (2002).

22. Levy, D. et al. Two-dimensional crystallization on lipid layer: a successful approach for membrane proteins. J. Struct. Biol. 127, 44-52 (1999).

23. Shimada, A. et al. Curved EFC/F-BAR-domain dimers are joined end to end into a filament for membrane invagination in endocytosis. Cell 129, 761-772 (2007).

24. Pykalainen, A. et al. Pinkbar is an epithelial-specific BAR domain protein that generates planar membrane structures. Nat. Struct. Mol. Biol. 18, 902-907 (2011).

25. Razzaq, A. et al. Amphiphysin is necessary for organization of the excitationcontraction coupling machinery of muscles, but not for synaptic vesicle endocytosis in Drosophila. Genes Dev. 15, 2967-2979 (2001).

26. Peter, B. J. et al. BAR domains as sensors of membrane curvature: the amphiphysin BAR structure. Science 303, 495-499 (2004).

27. Wang, Q. et al. Molecular mechanism of membrane constriction and tubulation mediated by the F-BAR protein Pacsin/Syndapin. Proc. Natl Acad. Sci. USA 106, 12700-12705 (2009).

28. Shimada, A. et al. Mapping of the basic amino-acid residues responsible for tubulation and cellular protrusion by the EFC/F-BAR domain of pacsin2/ Syndapin II. FEBS Lett. 584, 1111-1118 (2010).

29. Masuda, M. \& Mochizuki, N. Structural characteristics of BAR domain superfamily to sculpt the membrane. Semin. Cell Dev. Biol. 21, 391-398 (2010).

30. Kelley, C. F. et al. Membrane charge directs the outcome of F-BAR domain lipid binding and autoregulation. Cell Rep. 13, 2597-2609 (2015).

31. Ezekowitz, R. A., Sim, R. B., Hill, M. \& Gordon, S. Local opsonization by secreted macrophage complement components. Role of receptors for complement in uptake of zymosan. J. Exp. Med. 159, 244-260 (1984)

32. Itano, M. S. et al. Super-resolution imaging of C-type lectin spatial rearrangement within the dendritic cell plasma membrane at fungal microbe contact sites. Front. Phys. 2, https://doi.org/10.3389/fphy.2014.00046 (2014).

33. Paez-Segala, M. G. et al. Fixation-resistant photoactivatable fluorescent proteins for CLEM. Nat. Methods 12, 215-218 (2015).

34. Bigay, J. \& Antonny, B. Curvature, lipid packing, and electrostatics of membrane organelles: defining cellular territories in determining specificity. Dev. Cell 23, 886-895 (2012).

35. Suetsugu, S. et al. Optimization of WAVE2 complex-induced actin polymerization by membrane-bound IRSp53, PIP(3), and Rac. J. Cell Biol. 173, 571-585 (2006).

36. Saengsawang, W. et al. The F-BAR protein CIP4 inhibits neurite formation by producing lamellipodial protrusions. Curr. Biol. 22, 494-501 (2012).

37. Tsujita, K., Takenawa, T. \& Itoh, T. Feedback regulation between plasma membrane tension and membrane-bending proteins organizes cell polarity during leading edge formation. Nat. Cell Biol. 17, 749-758 (2015).

38. Guerrier, S. et al. The F-BAR domain of srGAP2 induces membrane protrusions required for neuronal migration and morphogenesis. Cell 138, 990-1004 (2009).

39. Almeida-Souza, L. et al. A flat BAR protein promotes actin polymerization at the base of clathrin-coated pits. Cell 174, 325-337 (2018).

40. Henne, W. M. et al. FCHo proteins are nucleators of clathrin-mediated endocytosis. Science 328, 1281-1284 (2010).

41. Castellano, F., Chavrier, P. \& Caron, E. Actin dynamics during phagocytosis. Semin. Immunol. 13, 347-355 (2001).

42. May, R. C. \& Machesky, L. M. Phagocytosis and the actin cytoskeleton. J. Cell Sci. 114, 1061-1077 (2001).

43. Insall, R. H. \& Machesky, L. M. Actin dynamics at the leading edge: from simple machinery to complex networks. Dev. Cell 17, 310-322 (2009).

44. Tsuboi, S. et al. FBP17 mediates a common molecular step in the formation of podosomes and phagocytic cups in macrophages. J. Biol. Chem. 284, 8548-8556 (2009).

45. Linkner, J. et al. The inverse BAR domain protein IBARa drives membrane remodeling to control osmoregulation, phagocytosis and cytokinesis. J. Cell Sci. 127, 1279-1292 (2014).

46. Sanchez-Barrena, M. J. et al. Bin2 is a membrane sculpting N-BAR protein that influences leucocyte podosomes, motility and phagocytosis. PLOS ONE 7, e52401 (2012).
47. Gallop, J. L., Walrant, A., Cantley, L. C. \& Kirschner, M. W. Phosphoinositides and membrane curvature switch the mode of actin polymerization via selective recruitment of toca-1 and Snx9. Proc. Natl Acad. Sci. USA 110 7193-7198 (2013).

48. Daste, F. et al. Control of actin polymerization via the coincidence of phosphoinositides and high membrane curvature. J. Cell Biol. 216, 3745-3765 (2017).

49. Takano, K., Toyooka, K. \& Suetsugu, S. EFC/F-BAR proteins and the NWASP-WIP complex induce membrane curvature-dependent actin polymerization. EMBO J. 27, 2817-2828 (2008).

50. May, R. C., Caron, E., Hall, A. \& Machesky, L. M. Involvement of the Arp $2 / 3$ complex in phagocytosis mediated by FcgammaR or CR3. Nat. Cell Biol. 2, 246-248 (2000).

51. Li, P. et al. Phase transitions in the assembly of multivalent signalling proteins. Nature 483, 336-340 (2012).

52. Senju, Y., Itoh, Y., Takano, K., Hamada, S. \& Suetsugu, S. Essential role of PACSIN2/syndapin-II in caveolae membrane sculpting. J. Cell Sci. 124, 2032-2040 (2011).

53. Kabsch, W. XDS. Acta Crystallogr. D 66, 125-132 (2010).

54. Otwinowski, Z. \& Minor, W. Processing of X-ray diffraction data collected in oscillation mode. Methods Enzymol. 276, 307-326 (1997).

55. Vonrhein, C., Blanc, E., Roversi, P. \& Bricogne, G. in Macromolecular Crystallography Protocols: Volume 2: Structure Determination (ed. Sylvie Doublié) 215-230 (Humana Press, Totowa, 2007).

56. Adams, P. D. et al. PHENIX: building new software for automated crystallographic structure determination. Acta Crystallogr. D 58, 1948-1954 (2002).

57. Berendsen, H. J. C., van der Spoel, D. \& van Drunen, R. GROMACS: a message-passing parallel molecular dynamics implementation. Comput. Phys. Commun. 91, 43-56 (1995).

58. Abraham, M. J. et al. GROMACS: high performance molecular simulations through multi-level parallelism from laptops to supercomputers. SoftwareX 12, 19-25 (2015).

59. Huang, J. et al. CHARMM36m: an improved force field for folded and intrinsically disordered proteins. Nat. Methods 14, 71-73 (2017).

60. Senju, Y. et al. Phosphorylation of PACSIN2 by protein kinase $\mathrm{C}$ triggers the removal of caveolae from the plasma membrane. J. Cell Sci. 128, 2766-2780 (2015).

61. Nagai, T. et al. A variant of yellow fluorescent protein with fast and efficient maturation for cell-biological applications. Nat. Biotechnol. 20, 87-90 (2002).

62. Mashiko, D. et al. Generation of mutant mice by pronuclear injection of circular plasmid expressing Cas9 and single guided. RNA Sci. Rep. 3, 3355 (2013).

63. Hsu, P. D. et al. DNA targeting specificity of RNA-guided Cas9 nucleases. Nat. Biotechnol. 31, 827-832 (2013).

64. Kitamura, T. et al. Retrovirus-mediated gene transfer and expression cloning: powerful tools in functional genomics. Exp. Hematol. 31, 1007-1014 (2003).

65. Nishimura, T. et al. DAAM1 stabilizes epithelial junctions by restraining WAVE complex-dependent lateral membrane motility. J. Cell Biol. 215, 559-573 (2016).

66. Wang, S., Moffitt, J. R., Dempsey, G. T., Xie, X. S. \& Zhuang, X. Characterization and development of photoactivatable fluorescent proteins for single-molecule-based superresolution imaging. Proc. Natl. Acad. Sci. USA 11, 8452-8457 (2014).

67. Fricke, F., Beaudouin, J., Eils, R. \& Heilemann, M. One, two or three? Probing the stoichiometry of membrane proteins by single-molecule localization microscopy. Sci. Rep. 5, 14072 (2015).

68. Berardozzi, R., Adam, V., Martins, A. \& Bourgeois, D. Arginine 66 controls dark-state formation in green-to-red photoconvertible fluorescent proteins. J. Am. Chem. Soc. 138, 558-565 (2016).

69. Ovesny, M., Krizek, P., Borkovec, J., Svindrych, Z. \& Hagen, G. M. ThunderSTORM: a comprehensive ImageJ plug-in for PALM and STORM data analysis and super-resolution imaging. Bioinformatics (Oxf., Engl.) 30, 2389-2390 (2014).

70. Ripley, B. D. The second-order analysis of stationary point processes. J. Appl. Probab. 13, 255-266 (1976).

\section{Acknowledgements}

We thank Prof. Oliver Daumke (Max-Delbrück-Centrum for Molecular Medicine) for critical reading of the manuscript. We thank Prof. Toshio Kitamura (The University of Tokyo) for the PLAT-A cells, Prof. Masahito Ikawa (Osaka University) and Prof. Taro Kawai (Nara Institute of Science and Technology) for the CRISPR/Cas9 system, Dr. Yohei Katoh and Prof. Kazuhisa Nakayama (Kyoto University) for the nanobody to GFP, Dr. Takahiro Fujiwara, Dr. Rinshi S. Kasai (Kyoto University) and Dr. Kenichi G.N. Suzuki (Gifu University) for advice on STORM and PALM, Dr. Kouta Mayanagi (Kyushu University), Dr. Hideki Shigematsu (RIKEN SPring-8 Center) and Ryo Ugawa (Laboratory for Technical Support, Medical Institute of Bioregulation, Kyushu University) for their support and advice on electron microscopy and Dr. Arthur Melo, 
Ayumi Takemoto, Kuniko Ohtake, Kosaku Kamihara, Ayana Wada, Katsuya Inamine, Yuki Kawasaki, Seiichiro Hayashi and all of the members of the laboratories for technical assistance and helpful discussions, and the staff members of the beamlines, Dr. Kunio Hirata (RIKEN SPring-8 Center), Dr. Naoki Sakai (RIKEN SPring-8 Center), Dr. Seiki Baba (Japan Synchrotron Radiation Research Institute) and Dr. Takashi Kumasaka (Japan Synchrotron Radiation Research Institute) and others, for assistance with our data collection. Synchrotron radiation experiments were performed at the Photon Factory with the approval of the Photon Factory Program Advisory Committee (Proposal Nos. 2011G092 and 2012G691) and at SPring-8 beamlines BL32XU, BL38B1 and BL44XU. This work was supported by grants from the Funding Program for Next Generation World-Leading Researchers (NEXT program LS031), JSPS (KAKENHI 26291037, JP15H0164, JP15H05902, JP17H03674, JP17H06006), JST CREST (JPMJCR1863), Osaka Cancer Research Foundation, the Naito Foundation, the Sumitomo Foundation, the Mitsubishi Foundation, the Sagawa Foundation for Promotion of Cancer Research and the NAIST Interdisciplinary Frontier Research Project to S.S., JSPS (KAKENHI 24687014, 25121726, JP17K07309) to A.S., JSPS (KAKENHI JP16K07351) to K.H.-S., JSPS (KAKENHI 23121507) to Y.I., JSPS (KAKENHI JP15H04357, JP17KT0026), MEXT 'Priority Issue on Post-K Computer' (Building Innovative Drug Discovery Infrastructure through Functional Control of Biomolecular Systems) to A.K. and Platform Project for Supporting Drug Discovery and Life Science Research (Basis for Supporting Innovative Drug Discovery and Life Science Research (BINDS)) from Japan Agency for Medical Research and Development (AMED), under Grant Numbers JP19am0101070 and JP19am0101072. The computations were partly performed using the supercomputers at the RCCS, The National Institute of Natural Science, ISSP and The University of Tokyo. This research also used the computational resources of the $\mathrm{K}$ computer, provided by the RIKEN Advanced Institute for Computational Science through the HPCI System Research project (Project ID: hp180201). This work was partly performed in the Cooperative Research Project Program of the Medical Institute of Bioregulation, Kyushu University.

\section{Author contributions}

K.H.-S., Y.I., K.Takeshita and A.S. collected the X-ray diffraction data and determined the crystal structures. K.Takemura and A.K. performed MD simulations. K.H.-S., S.K., M.A.F., W.N.I.W.M.N., T.H.N.T., T.I. and K.Takeshita, expressed the proteins and performed biochemical analyses. N.M., M.A.F., K.I., S.S. and A.S. performed the electron micrographic observations. T.N., S.H.-N., K.O.-Y. and S.S. performed the cell biological analyses. K.H.-S., K.O.-Y., M.T. and S.S. prepared and analysed the super-resolution imaging samples. S.S., A.K., D.K., M.Y. and A.S. designed the research and supervised the project. K.H.-S., Y.I., M.A.F., T.N., K.Takemura, A.K., A.S. and S.S. wrote the manuscript with input from all other authors.

\section{Competing interests}

The authors declare no competing interests.

\section{Additional information}

Supplementary information is available for this paper at https://doi.org/10.1038/s41467 019-12738-w.

Correspondence and requests for materials should be addressed to A.S. or S.S.

Peer review information Nature Communications thanks the anonymous reviewer(s) for their contribution to the peer review of this work. Peer reviewer reports are available.

Reprints and permission information is available at http://www.nature.com/reprints

Publisher's note Springer Nature remains neutral with regard to jurisdictional claims in published maps and institutional affiliations.

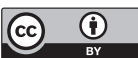

Open Access This article is licensed under a Creative Commons Attribution 4.0 International License, which permits use, sharing, adaptation, distribution and reproduction in any medium or format, as long as you give appropriate credit to the original author(s) and the source, provide a link to the Creative Commons license, and indicate if changes were made. The images or other third party material in this article are included in the article's Creative Commons license, unless indicated otherwise in a credit line to the material. If material is not included in the article's Creative Commons license and your intended use is not permitted by statutory regulation or exceeds the permitted use, you will need to obtain permission directly from the copyright holder. To view a copy of this license, visit http://creativecommons.org/ licenses/by/4.0/.

(C) The Author(s) 2019 